\title{
St. Vincent and the Grenadines: Statistical Appendix
}

This Statistical Appendix for St. Vincent and the Grenadines was prepared by a staff team of the International Monetary Fund as background documentation for the periodic consultation with the member country. It is based on the information available at the time it was completed on January 14, 2008. The views expressed in this document are those of the staff team and do not necessarily reflect the views of the Government of St. Vincent and the Grenadines or the Executive Board of the IMF.

The policy of publication of staff reports and other documents by the IMF allows for the deletion of market-sensitive information.

\footnotetext{
Copies of this report are available to the public from

International Monetary Fund $\bullet$ Publication Services

700 19th Street, N.W. • Washington, D.C. 20431

Telephone: (202) 623-7430 • Telefax: (202) 623-7201

E-mail: publications@imf.org • Internet: http://www.imf.org
}

\section{International Monetary Fund \\ Washington, D.C.}





\section{INTERNATIONAL MONETARY FUND}

ST. VINCENT AND THE GRENADINES

\section{Statistical Appendix}

Prepared by a staff team consisting of Catherine Pattillo, Emilio Pineda, Evridiki Tsounta (all WHD), and Ritu Basu (FIN)

Approved by Western Hemisphere Department

January 14,2008

Contents

Basic Data 3

Statistical Appendix Tables

I. Real Sector

1. Selected Price and Production Indicators ………….....................................................

2. GDP by Type of Expenditure at Current Prices.............................................................6

3. GDP by Economic Activity at Current Factor Cost........................................................

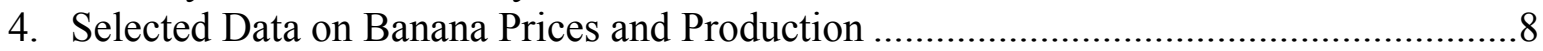

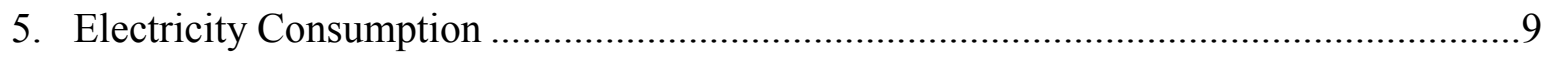

6. Petroleum Consumption and Unit Values................................................................

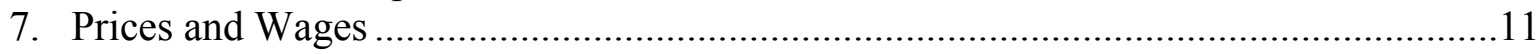

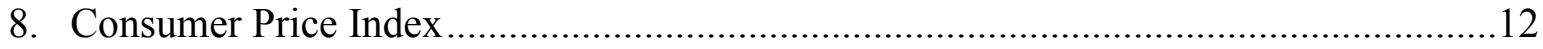

II. Fiscal Sector

9. Summary of Consolidated Public Sector Operations....................................................13

10. Summary of Central Government Operations ................................................................14

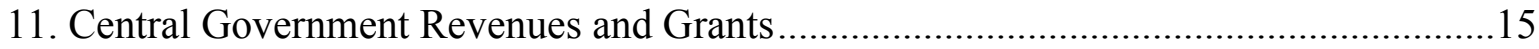

12. Central Government Expenditure and Net Lending ......................................................16

13. Central Government Investment Plan (CGIP) ……….................................................17

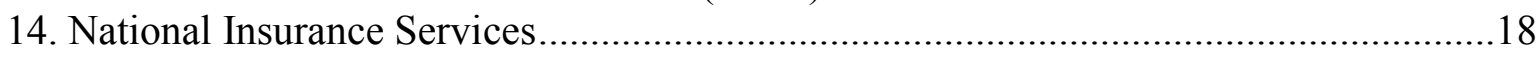

15. Public Enterprise Operations (Including Kingstown Board) .........................................19

16. Operations of Selected Public Enterprises ........................................................................20 


\section{Monetary Sector}

17. Monetary Survey

18. Eastern Caribbean Central Bank Operations ............................................................22

19. Consolidated Balance Sheet of Commercial Banks......................................................23

20. Balance Sheet of Local Commercial Banks................................................................24

21. Consolidated Balance Sheet of Foreign Commercial Banks .....................................25

22. Distribution of Commercial Bank Lending ...........................................................26

23. Interest Rate Structure of Commercial Banks ........................................................27

\section{External Sector}

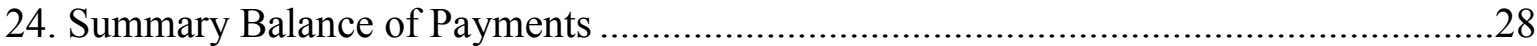

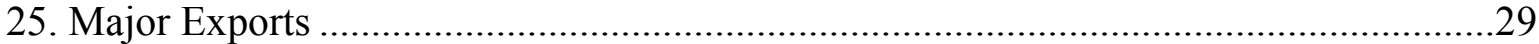

26. Value, Volume, and Unit Value of Major Agricultural Exports.................................30

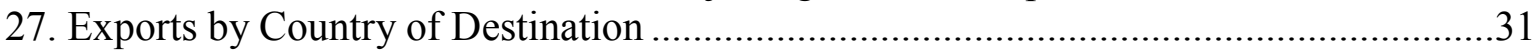

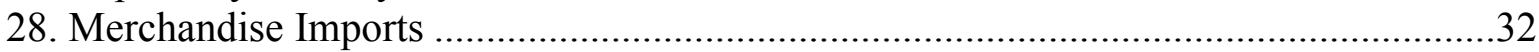

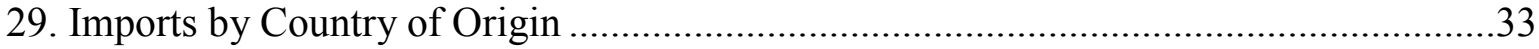

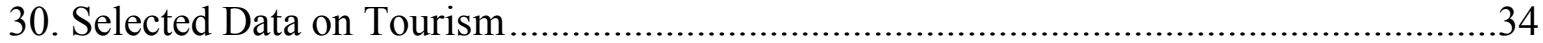

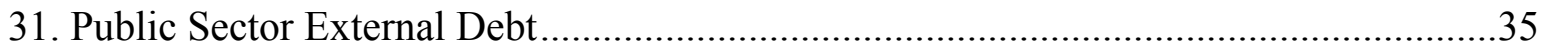




\section{St. Vincent and the Grenadines: Basic Data}

I. Social and Demographic Indicators

Area (sq. km)

Population

Total (thousands, 2006)

Rate of growth (percent per year, 2006)

Density (per sq. km., 2006)

Population characteristics (2004)

Life expectancy at birth (years)

Infant mortality (per thousand live births)

Under 5 mortality rate (per thousand)
389

0.01

274.6

71.3

18

22

Adult illiteracy rate (percent, 2001)

Health and nutrition

Calorie intake (per capita a day, 2004)

Population per physician (thousand, 1997)

2,660

AIDS incidence rate (per 100,000, 2004)

Gross domestic product (2006)

(millions of US dollars)

(millions of EC dollars)

(US\$ per capita)

II. Economic Indicators, 2002-06

\begin{tabular}{|c|c|c|c|c|c|}
\hline & 2002 & 2003 & 2004 & 2005 & $\begin{array}{r}\text { Est. } \\
2006 \\
\end{array}$ \\
\hline & \multicolumn{5}{|c|}{ (In percent of GDP at factor cost) } \\
\hline \multicolumn{6}{|l|}{ Origin of GDP } \\
\hline Agriculture and mining & 11.9 & 11.3 & 10.1 & 9.5 & 9.5 \\
\hline Manufacturing and construction & 14.9 & 15.3 & 15.8 & 15.5 & 15.7 \\
\hline Electricity and water & 6.8 & 7.1 & 7.2 & 7.6 & 7.2 \\
\hline \multirow[t]{2}{*}{ Services } & 66.3 & 66.2 & 67.0 & 67.4 & 67.5 \\
\hline & \multicolumn{5}{|c|}{ (Annual percentage change, unless otherwise stated) } \\
\hline Real GDP (at factor cost) & 3.2 & 2.8 & 6.8 & 2.6 & 6.9 \\
\hline Real GDP per capita & 3.1 & 2.7 & 6.7 & 2.5 & 6.8 \\
\hline GDP deflator & 2.0 & 1.4 & 2.0 & 3.7 & 3.6 \\
\hline Consumer prices (annual average) & 0.8 & 0.2 & 3.0 & 3.7 & 3.0 \\
\hline \multirow[t]{2}{*}{ Consumer prices (end of period) } & 0.4 & 2.7 & 1.7 & 3.9 & 4.8 \\
\hline & \multicolumn{5}{|c|}{ (In percent of GDP) } \\
\hline \multicolumn{6}{|l|}{ National account } \\
\hline Gross domestic investment & 29.7 & 33.3 & 32.2 & 32.2 & 35.4 \\
\hline \multicolumn{6}{|l|}{ Of which } \\
\hline Public investment & 10.0 & 10.0 & 12.9 & 12.6 & 14.3 \\
\hline Gross national savings & 18.2 & 12.5 & 7.4 & 9.5 & 11.0 \\
\hline External savings & 11.5 & 20.8 & 24.8 & 22.7 & 24.4 \\
\hline Private consumption & 59.8 & 65.1 & 69.2 & 68.7 & 69.4 \\
\hline Public consumption & 20.5 & 19.5 & 19.8 & 19.8 & 18.8 \\
\hline \multicolumn{6}{|l|}{ Public finances } \\
\hline \multicolumn{6}{|l|}{ Central government } \\
\hline Total revenue and grants & 31.7 & 31.3 & 29.6 & 30.0 & 30.8 \\
\hline Current revenue & 30.6 & 30.8 & 28.8 & 28.5 & 30.0 \\
\hline Total expenditure & 34.0 & 34.7 & 33.1 & 35.5 & 35.7 \\
\hline Current expenditure & 28.0 & 26.7 & 26.0 & 27.3 & 26.8 \\
\hline \multicolumn{6}{|l|}{ Of which } \\
\hline Interest & 2.6 & 2.7 & 2.5 & 3.0 & 3.2 \\
\hline Capital expenditure & 6.4 & 7.9 & 7.1 & 8.2 & 8.9 \\
\hline Savings & 2.6 & 4.1 & 2.9 & 1.2 & 3.2 \\
\hline Primary balance & -1.6 & -0.6 & -1.1 & -2.5 & -1.7 \\
\hline Overall balance & -4.2 & -3.3 & -3.5 & -5.5 & -4.9 \\
\hline \multicolumn{6}{|l|}{ Consolidated public sector } \\
\hline Current balance & 5.8 & 6.5 & 5.3 & 3.4 & 5.5 \\
\hline Capital expenditure & 10.0 & 10.0 & 12.9 & 12.6 & 14.3 \\
\hline Overall balance & -5.9 & -3.0 & -6.8 & -7.7 & -6.3 \\
\hline
\end{tabular}


St. Vincent and the Grenadines: Basic Data (continued)

\begin{tabular}{|c|c|c|c|c|c|}
\hline & 2002 & 2003 & 2004 & 2005 & $\begin{array}{r}\text { Est. } \\
2006 \\
\end{array}$ \\
\hline & \multicolumn{5}{|c|}{$\begin{array}{l}\text { (Percent contribution to growth in broad money, } \\
\text { unless otherwise indicated) } 1 /\end{array}$} \\
\hline \multicolumn{6}{|l|}{ Money and credit } \\
\hline Liabilities to the private sector (broad money) & 8.3 & 1.9 & 13.7 & 6.4 & 7.5 \\
\hline \multicolumn{6}{|l|}{ Of which } \\
\hline Money & 9.1 & 6.1 & 13.1 & 14.2 & 5.1 \\
\hline Quasi money & 7.9 & -0.3 & 14.0 & 2.2 & 9.0 \\
\hline Net domestic assets & 5.4 & -3.9 & -2.0 & 11.0 & 3.0 \\
\hline Net credit to the public sector & 4.8 & -4.0 & 2.7 & 7.5 & 0.1 \\
\hline Credit to the private sector & 0.9 & 0.6 & 0.8 & 4.3 & 11.1 \\
\hline Other assets (net) & -4.6 & -0.2 & -10.0 & 0.3 & -9.2 \\
\hline Liabilities to the private sector (in percent of GDP) & 71.9 & 70.0 & 73.5 & 74.0 & 70.6 \\
\hline & \multicolumn{5}{|c|}{ (In millions of U.S. dollars, unless otherwise stated) } \\
\hline \multicolumn{6}{|l|}{ Balance of payments } \\
\hline Current account balance & -42.0 & -79.5 & -102.5 & -99.3 & -120.1 \\
\hline Merchandise trade balance & -116.5 & -136.7 & -159.7 & -169.8 & -198.5 \\
\hline Exports & 41.2 & 40.1 & 39.3 & 42.6 & 41.2 \\
\hline Imports & 157.8 & 176.8 & 199.0 & 212.4 & 239.7 \\
\hline Service balance & 80.0 & 68.2 & 72.0 & 79.2 & 82.4 \\
\hline Capital and financial account & 25.9 & 63.4 & 111.8 & 71.0 & 132.2 \\
\hline Errors and omissions & 9.4 & 15.6 & 16.1 & 25.4 & 0.0 \\
\hline Exports of goods and nonfactor services (in percent of GDP) & 48.8 & 45.2 & 44.6 & 45.8 & 43.0 \\
\hline Imports of goods and nonfactor services (in percent of GDP) & 58.8 & 63.1 & 65.7 & 66.5 & 66.6 \\
\hline Current account (in percent of GDP) & -11.5 & -20.8 & -24.8 & -22.7 & -24.4 \\
\hline Terms of trade (annual percentage change) & 8.2 & -0.9 & -8.6 & -3.0 & -5.7 \\
\hline Real effective exchange rate (e.o.p. percentage change) & -2.0 & -7.3 & -3.6 & 0.1 & -0.6 \\
\hline \multicolumn{6}{|l|}{ Public debt } \\
\hline Total public sector debt in percent of GDP & 70.5 & 72.3 & 77.0 & 80.7 & 78.0 \\
\hline Outstanding public external debt in percent of GDP & 46.5 & 50.9 & 53.3 & 53.8 & 48.0 \\
\hline $\begin{array}{l}\text { External public debt service (in percent of exports of goods and service! } \\
\text { Of which }\end{array}$ & 6.3 & 7.1 & 9.8 & 10.7 & 11.8 \\
\hline Interest & 2.6 & 2.5 & 3.1 & 3.9 & 0.0 \\
\hline \multicolumn{6}{|l|}{ IMF data (as of December 31,2007 ) } \\
\hline Membership status: & & & & \multicolumn{2}{|c|}{ Article VIII status } \\
\hline Intervention currency and rate & & & & \multicolumn{2}{|c|}{ EC $\$ 2.7$ per US\$ } \\
\hline Quota & & & & \multicolumn{2}{|c|}{ SDR 8.3 million } \\
\hline Fund holdings of local currency & & & & \multicolumn{2}{|c|}{ SDR 7.8 million } \\
\hline As percent of quota & & & & \multicolumn{2}{|r|}{94.0} \\
\hline Outstanding purchases and loans & & & & \multicolumn{2}{|r|}{0.01} \\
\hline \multicolumn{6}{|l|}{ Special Drawing Rights Department } \\
\hline Cumulative SDR allocation & & & & \multicolumn{2}{|c|}{ SDR 0.35 million } \\
\hline Holdings of SDRs & & & & \multicolumn{2}{|c|}{ SDR 0.01 million } \\
\hline
\end{tabular}

Sources: St. Vincent and the Grenadines authorities; Eastern Caribbean Central Bank (ECCB); CIA World Factbook; World Bank; and Fund staff estimates.

$1 /$ In relation to banking system liabilities to the private sector at the beginning of the period. 
Table 1. St. Vincent and the Grenadines: Selected Price and Production Indicators

\begin{tabular}{|c|c|c|c|c|c|}
\hline & 2002 & 2003 & 2004 & 2005 & $\begin{array}{r}\text { Est. } \\
2006 \\
\end{array}$ \\
\hline & \multicolumn{5}{|c|}{ (In millions of Eastern Caribbean dollars) } \\
\hline Nominal GDP (at market prices) & 986 & 1,032 & 1,118 & 1,182 & 1,330 \\
\hline \multirow[t]{2}{*}{ Real GDP (at factor cost) } & 632 & 650 & 694 & 712 & 760 \\
\hline & \multicolumn{5}{|c|}{ (Annual percentage change) } \\
\hline Nominal GDP (at market prices) & 5.7 & 4.7 & 8.3 & 5.7 & 12.6 \\
\hline Real GDP (at factor cost) & 3.2 & 2.8 & 6.8 & 2.6 & 6.9 \\
\hline Agriculture, forestry and fishing & 7.8 & -2.2 & -5.2 & -3.9 & 7.6 \\
\hline Manufacturing & -6.1 & -2.9 & 3.3 & 1.6 & 2.9 \\
\hline Electricity and water & 2.6 & 4.0 & 7.1 & 8.4 & 1.7 \\
\hline Construction & -2.9 & 6.0 & 14.7 & 0.4 & 11.4 \\
\hline Wholesale and retail trade & 3.1 & 3.5 & 9.8 & 4.4 & 6.8 \\
\hline Hotels and restaurants & -8.0 & 2.0 & 7.3 & 9.5 & 1.0 \\
\hline Transport & 6.2 & 2.0 & 10.1 & -0.7 & 18.0 \\
\hline Communications & 4.5 & 4.0 & 19.3 & -1.1 & 2.0 \\
\hline Banks and insurance & 10.1 & 4.0 & 3.1 & 10.4 & 5.8 \\
\hline Real estate and housing & 1.5 & 1.5 & 1.5 & 1.5 & 2.0 \\
\hline Government services & 3.7 & 0.1 & 2.5 & 5.3 & 4.3 \\
\hline GDP deflator (at factor cost) & 2.0 & 1.4 & 2.0 & 3.7 & 3.6 \\
\hline Consumer prices (end of period) & 0.4 & 2.7 & 1.7 & 3.9 & 4.8 \\
\hline Consumer prices (period average) & 0.8 & 0.2 & 3.0 & 3.7 & 3.0 \\
\hline
\end{tabular}

Sources: Ministry of Finance and Planning; and Fund staff estimates. 
Table 2. St. Vincent and the Grenadines: GDP by Type of Expenditure at Current Prices

\begin{tabular}{|c|c|c|c|c|c|}
\hline & 2002 & 2003 & 2004 & 2005 & $\begin{array}{r}\text { Est. } \\
2006\end{array}$ \\
\hline & \multicolumn{5}{|c|}{ (In millions of Eastern Caribbean dollars) } \\
\hline Consumption expenditure & 792 & 874 & 995 & 1,046 & 1,173 \\
\hline Private sector & 590 & 672 & 774 & 812 & 923 \\
\hline Public sector & 202 & 202 & 221 & 234 & 250 \\
\hline Gross capital formation & 293 & 344 & 360 & 381 & 471 \\
\hline Public sector & 99 & 104 & 144 & 149 & 191 \\
\hline Private sector & 194 & 240 & 216 & 231 & 280 \\
\hline Gross domestic expenditure & 1,085 & 1,218 & 1,355 & 1,426 & 1,644 \\
\hline Foreign balance & -113 & -215 & -277 & -268 & -324 \\
\hline Exports of goods and nonfactor services & 481 & 467 & 498 & 541 & 572 \\
\hline Export of goods & 111 & 108 & 106 & 115 & 111 \\
\hline Export of nonfactor services & 370 & 358 & 392 & 427 & 461 \\
\hline Imports of goods and nonfactor services & -580 & -652 & -735 & -786 & -886 \\
\hline Import of goods & -426 & -477 & -537 & -573 & -647 \\
\hline Import of nonfactor services & -154 & -174 & -198 & -213 & -239 \\
\hline \multirow[t]{2}{*}{ Nominal GDP (at market prices) } & 986 & 1,032 & 1,118 & 1,182 & 1,330 \\
\hline & \multicolumn{5}{|c|}{ (In percent of nominal GDP) } \\
\hline Consumption expenditure & 80.3 & 84.6 & 89.0 & 88.5 & 88.2 \\
\hline Private sector & 59.8 & 65.1 & 69.2 & 68.7 & 69.4 \\
\hline Public sector & 20.5 & 19.5 & 19.8 & 19.8 & 18.8 \\
\hline Gross capital formation & 29.7 & 33.3 & 32.2 & 32.2 & 35.4 \\
\hline Public sector & 10.0 & 10.0 & 12.9 & 12.6 & 14.3 \\
\hline Private sector & 19.7 & 23.3 & 19.3 & 19.6 & 21.1 \\
\hline Gross domestic expenditure & 110.0 & 117.9 & 121.2 & 120.7 & 123.6 \\
\hline Foreign balance & -11.5 & -20.8 & -24.8 & -22.7 & -24.4 \\
\hline Exports of goods and nonfactor services & 48.8 & 45.2 & 44.6 & 45.8 & 43.0 \\
\hline Imports of goods and nonfactor services & -58.8 & -63.1 & -65.7 & -66.5 & -66.6 \\
\hline \multirow[t]{2}{*}{ Nominal GDP (at market prices) } & 100.0 & 100.0 & 100.0 & 100.0 & 100.0 \\
\hline & \multicolumn{5}{|c|}{ (Annual percentage change) } \\
\hline Consumption expenditure & 1.2 & 10.3 & 13.9 & 5.1 & 12.2 \\
\hline Private sector & -1.2 & 13.9 & 15.1 & 4.9 & 13.8 \\
\hline Public sector & 8.9 & -0.2 & 9.7 & 5.9 & 6.7 \\
\hline Gross fixed capital & 18.5 & 17.5 & 4.7 & 5.7 & 23.6 \\
\hline Public sector & 22.3 & 4.7 & 39.1 & 3.5 & 27.8 \\
\hline Private sector & 16.6 & 24.0 & -10.2 & 7.2 & 21.0 \\
\hline Gross domestic expenditure & 5.3 & 12.2 & 11.3 & 5.3 & 15.2 \\
\hline Foreign balance & 1.6 & 87.8 & 28.0 & 3.4 & 28.1 \\
\hline Exports of goods and nonfactor services & 1.4 & -3.1 & 6.7 & 8.7 & 5.7 \\
\hline Imports of goods and nonfactor services & 3.3 & 12.4 & 12.8 & 7.0 & 12.7 \\
\hline Nominal GDP (at market prices) & 5.7 & 4.7 & 8.3 & 5.7 & 12.6 \\
\hline
\end{tabular}

Sources: Ministry of Finance and Planning; and Fund staff estimates. 
Table 3. St. Vincent and the Grenadines: GDP by Economic Activity at Current Factor Cost

\begin{tabular}{|c|c|c|c|c|c|}
\hline & 2002 & 2003 & 2004 & 2005 & $\begin{array}{r}\text { Est. } \\
2006 \\
\end{array}$ \\
\hline & \multicolumn{5}{|c|}{ (In millions of Eastern Caribbean dollars) } \\
\hline Primary sector & 82 & 77 & 78 & 84 & 87 \\
\hline Agriculture & 81 & 75 & 76 & 81 & 84 \\
\hline Banana & 24 & 14 & 16 & 15 & 13 \\
\hline Other crops & 38 & 39 & 39 & 46 & 49 \\
\hline Livestock & 6 & 6 & 6 & 6 & 6 \\
\hline Forestry & 5 & 5 & 5 & 6 & 6 \\
\hline Fishing & 8 & 10 & 10 & 8 & 9 \\
\hline Mining and quarrying & 2 & 2 & 2 & 2 & 2 \\
\hline Secondary sector & 197 & 207 & 226 & 234 & 274 \\
\hline Manufacturing & 55 & 50 & 54 & 59 & 63 \\
\hline Electricity and water & 50 & 56 & 55 & 55 & 56 \\
\hline Construction & 93 & 101 & 117 & 120 & 155 \\
\hline Tertiary sector & 538 & 569 & 624 & 670 & 733 \\
\hline Wholesale and retail trade & 150 & 157 & 177 & 192 & 211 \\
\hline Hotels and restaurants & 17 & 19 & 21 & 23 & 23 \\
\hline Transport & 112 & 118 & 131 & 133 & 157 \\
\hline Communications & 50 & 49 & 59 & 56 & 57 \\
\hline Banks and insurance & 63 & 81 & 93 & 116 & 123 \\
\hline Real estate and housing & 19 & 20 & 21 & 21 & 22 \\
\hline Government services & 163 & 162 & 172 & 187 & 204 \\
\hline Other services & 16 & 18 & 19 & 21 & 22 \\
\hline Less: imputed service charge & 52 & 55 & 67 & 78 & 85 \\
\hline \multirow[t]{2}{*}{ Nominal GDP at current factor cost } & 817 & 852 & 928 & 988 & 1,094 \\
\hline & \multicolumn{5}{|c|}{ (In percent of GDP) } \\
\hline Primary sector & 10.1 & 9.0 & 8.4 & 8.5 & 7.9 \\
\hline Agriculture & 9.9 & 8.8 & 8.2 & 8.2 & 7.7 \\
\hline Banana & 2.9 & 1.7 & 1.7 & 1.5 & 1.2 \\
\hline Other crops & 4.6 & 4.6 & 4.2 & 4.7 & 4.5 \\
\hline Livestock & 0.7 & 0.7 & 0.7 & 0.6 & 0.6 \\
\hline Forestry & 0.7 & 0.6 & 0.6 & 0.6 & 0.5 \\
\hline Fishing & 1.0 & 1.2 & 1.0 & 0.9 & 0.9 \\
\hline Mining and quarrying & 0.2 & 0.2 & 0.2 & 0.2 & 0.2 \\
\hline Secondary sector & 24.1 & 24.2 & 24.3 & 23.7 & 25.1 \\
\hline Manufacturing & 6.7 & 5.8 & 5.8 & 6.0 & 5.8 \\
\hline Electricity and water & 6.1 & 6.6 & 5.9 & 5.6 & 5.1 \\
\hline Construction & 11.3 & 11.8 & 12.6 & 12.1 & 14.2 \\
\hline Tertiary sector & 65.8 & 66.8 & 67.2 & 67.9 & 67.0 \\
\hline Wholesale and retail trade & 18.3 & 18.4 & 19.1 & 19.4 & 19.3 \\
\hline Hotels and restaurants & 2.1 & 2.3 & 2.2 & 2.3 & 2.1 \\
\hline Transport & 13.7 & 13.9 & 14.1 & 13.4 & 14.3 \\
\hline Communications & 6.1 & 5.7 & 6.3 & 5.6 & 5.2 \\
\hline Banks and insurance & 7.7 & 9.5 & 10.0 & 11.7 & 11.2 \\
\hline Real estate and housing & 2.4 & 2.3 & 2.2 & 2.2 & 2.0 \\
\hline Government services & 19.9 & 19.0 & 18.6 & 19.0 & 18.6 \\
\hline Other services & 2.0 & 2.1 & 2.0 & 2.1 & 2.0 \\
\hline Less: imputed service charge & 6.3 & 6.4 & 7.2 & 7.9 & 7.8 \\
\hline Nominal GDP at market prices & 100.0 & 100.0 & 100.0 & 100.0 & 100.0 \\
\hline
\end{tabular}

Sources: Ministry of Finance and Planning; and Fund staff estimates. 
Table 4. St. Vincent and the Grenadines: Selected Data on Banana Prices and Production

\begin{tabular}{lrrrrr}
\hline & 2002 & 2003 & 2004 & 2005 & 2006 \\
\hline & 37,551 & 26,686 & 28,056 & 25,211 & 22,678 \\
Volume exported (metric tons) 1/ & & & & & \\
& 42,837 & 32,026 & 35,241 & 32,107 & 34,193 \\
Export earnings (thousands EC\$) 1/ & 15,866 & 11,861 & 13,052 & 11,891 & 12,664 \\
Export earnings (thousands US\$) & 8.9 & 6.9 & 7.1 & 5.9 & 6.0 \\
Percentage of exports of goods and services & & & & & \\
& 0.523 & 0.559 & 0.572 & 0.621 & 0.624 \\
Banana Growers' Association (BGA) price (EC\$/b) 2/ & 0.395 & 0.370 & 0.370 & 0.370 & 0.406 \\
Price received by growers (EC\$/lb) & & & & & \\
& 85 & 85 & 85 & 85 & 80 \\
Quality (Southnampton PUWS) 3/ & & & & & \\
& & $($ Annual percentage change) & \\
& 16.0 & -25.2 & 10.0 & -8.9 & 6.5 \\
Export earnings (EC\$) & 10.8 & -28.9 & 5.1 & -10.1 & -10.0 \\
Volume exported & 4.1 & 6.9 & 2.3 & 8.6 & 0.5 \\
BGA price (EC\$/lb) & & & & &
\end{tabular}

Sources: Banana Growers Association; FAO; and Fund staff estimates.

1/ Banana Growers Association only.

2/ Price received by the Banana Growers Association from WIBDECO.

$3 /$ Percentage of units within specification. 
Table 5. St. Vincent and the Grenadines: Electricity Consumption

\begin{tabular}{|c|c|c|c|c|c|}
\hline & 2002 & 2003 & 2004 & 2005 & $\begin{array}{r}\text { Est. } \\
2006 \\
\end{array}$ \\
\hline & \multicolumn{5}{|c|}{ (In thousands of kwh) } \\
\hline Total generated & 101,846 & 108,240 & 120,742 & 131,751 & 134,302 \\
\hline Commercial consumption & 36,571 & 39,004 & 47,087 & 53,541 & 54,135 \\
\hline Domestic consumption & 44,185 & 47,195 & 50,494 & 53,688 & 54,867 \\
\hline Industrial consumption & 6,500 & 6,520 & 6,147 & 6,309 & 6,587 \\
\hline Street lighting consumption & 2,569 & 2,711 & 2,796 & 2,881 & 2,937 \\
\hline Other consumption & 1,600 & 3,020 & 3,810 & 4,914 & 3,939 \\
\hline Transmission loss & 10,422 & 9,789 & 10,407 & 10,419 & 11,838 \\
\hline Number of consumers & 28,595 & 29,535 & 30,304 & 31,681 & 32,710 \\
\hline \multicolumn{6}{|l|}{ Electricity consumption } \\
\hline per consumer & 3.6 & 3.7 & 4.0 & 4.2 & 4.1 \\
\hline \multirow[t]{2}{*}{ Net generation } & 91,425 & 98,451 & 110,334 & 121,332 & 122,464 \\
\hline & \multicolumn{5}{|c|}{ (In percent of total) } \\
\hline Total generated & 100.0 & 100.0 & 100.0 & 100.0 & 100.0 \\
\hline Commercial & 35.9 & 36.0 & 39.0 & 40.6 & 40.3 \\
\hline Domestic & 43.4 & 43.6 & 41.8 & 40.7 & 40.9 \\
\hline Industrial & 6.4 & 6.0 & 5.1 & 4.8 & 4.9 \\
\hline Street lighting & 2.5 & 2.5 & 2.3 & 2.2 & 2.2 \\
\hline Other & 1.6 & 2.8 & 3.2 & 3.7 & 2.9 \\
\hline Transmission loss & 10.2 & 9.0 & 8.6 & 7.9 & 8.8 \\
\hline
\end{tabular}

Source: St. Vincent Electricity Services Limited (VINLEC). 
Table 6. St. Vincent and the Grenadines: Petroleum Consumption and Unit Values 1/

\begin{tabular}{|c|c|c|c|c|c|}
\hline & 2002 & 2003 & 2004 & 2005 & $\begin{aligned} \text { Est. } \\
2006\end{aligned}$ \\
\hline & \multicolumn{5}{|c|}{$\begin{array}{l}\text { (Value in thousands of Eastern Caribbean dollars, quantities in } \\
\text { cubic metres, and unit values in EC } \$ \text { per cubic meter) }\end{array}$} \\
\hline \multicolumn{6}{|l|}{ Gasoline } \\
\hline Value & 11,944 & 14,333 & 18,834 & 22,865 & 28,360 \\
\hline Cubic metre & 25,572 & 24,775 & 26,715 & 24,755 & 26,229 \\
\hline Unit values & 467 & 579 & 705 & 924 & 1,081 \\
\hline \multicolumn{6}{|l|}{ Diesel } \\
\hline Value & 20,751 & 29,560 & 36,242 & 52,515 & 62,774 \\
\hline Cubic metre & 42,511 & 49,720 & 54,627 & 48,731 & 54,696 \\
\hline Unit values & 488 & 595 & 663 & 1,078 & 1,148 \\
\hline \multicolumn{6}{|l|}{ Kerosene } \\
\hline Value & 227 & 231 & 208 & 416 & 390 \\
\hline Cubic metre & 518 & 444 & 288 & 400 & 336 \\
\hline Unit values & 438 & 520 & 722 & 1,040 & 1,161 \\
\hline \multicolumn{6}{|l|}{ Butane } \\
\hline Value & 5,118 & 5,699 & 7,644 & 8,930 & 9,041 \\
\hline Metric tonnes & 5,435 & 3,566 & 4,121 & 3,518 & 4,034 \\
\hline \multirow[t]{2}{*}{ Unit values } & 942 & 1,598 & 1,855 & 2,538 & 2,241 \\
\hline & \multicolumn{5}{|c|}{ (Annual percentage change of consumption) } \\
\hline Gasoline & 6.2 & -3.1 & 7.8 & -7.3 & 6.0 \\
\hline Diesel & 3.7 & 17.0 & 9.9 & -10.8 & 12.2 \\
\hline Kerosene & 8.1 & -14.3 & -35.1 & 38.9 & -16.0 \\
\hline Natural gas & 52.6 & -34.4 & 15.6 & -14.6 & 14.7 \\
\hline
\end{tabular}

Source: Ministry of Finance and Planning.

$1 /$ Prices at end of period. 
Table 7. St. Vincent and the Grenadines: Prices and Wages (Annual percentage change)

\begin{tabular}{|c|c|c|c|c|c|}
\hline & 2002 & 2003 & 2004 & 2005 & $\begin{array}{r}\text { Est. } \\
2006\end{array}$ \\
\hline & \multicolumn{5}{|c|}{ I. Consumer Prices 1/ } \\
\hline January & 1.9 & -0.8 & 3.4 & 1.7 & 3.4 \\
\hline February & 1.8 & -1.2 & 2.6 & 3.2 & 2.6 \\
\hline March & 1.7 & -1.9 & 4.1 & 3.1 & 2.2 \\
\hline April & 1.4 & -1.7 & 4.2 & 3.9 & 1.6 \\
\hline May & 0.2 & -0.2 & 3.1 & 4.5 & 2.4 \\
\hline June & 0.1 & 1.1 & 2.4 & 4.8 & 2.3 \\
\hline July & -0.7 & 1.6 & 2.4 & 4.8 & 2.7 \\
\hline August & 0.0 & 1.3 & 2.5 & 4.3 & 2.5 \\
\hline September & 1.4 & -0.8 & 3.8 & 3.1 & 4.5 \\
\hline October & 1.8 & 0.0 & 3.3 & 3.6 & 3.1 \\
\hline November & -0.3 & 2.8 & 2.0 & 3.8 & 4.3 \\
\hline December & 0.4 & 2.7 & 1.7 & 3.9 & 4.8 \\
\hline \multirow[t]{2}{*}{ Average } & 0.8 & 0.2 & 3.0 & 3.7 & 3.0 \\
\hline & \multicolumn{5}{|c|}{ II. Wages } \\
\hline Central government 2/ & 0.0 & 0.0 & 4.0 & 4.5 & 5.0 \\
\hline Public enterprises $3 /$ & 3.0 & 3.8 & $\ldots$ & 4.5 & 5.0 \\
\hline \multicolumn{6}{|l|}{ Private sector } \\
\hline Geest, and Cable and Wireless & 4.0 & 4.0 & $\ldots$ & $\ldots$ & $\ldots$ \\
\hline
\end{tabular}

Sources: Ministry of Finance and Planning; Labor Commission; Ministry of Agriculture, Industry, and Labor; and various public enterprises.

1/ The CPI is based on 2001.

2/ Central government wages were frozen in 2002 and 2003.

$3 /$ Wage adjustments in four major public enterprises. 
Table 8. St. Vincent and the Grenadines: Consumer Price Index

\begin{tabular}{|c|c|c|c|c|c|}
\hline & 2002 & 2003 & 2004 & 2005 & $\begin{array}{r}\text { Est. } \\
2006\end{array}$ \\
\hline & \multicolumn{5}{|c|}{ (Index January 2001=100) } \\
\hline End of period & 100.5 & 103.2 & 105.0 & 109.1 & 114.3 \\
\hline Food & 99.2 & 103.7 & 105.8 & 112.1 & 118.5 \\
\hline Alcoholic beverages and tobacco & 103.2 & 101.3 & 105.3 & 109.8 & 112.6 \\
\hline Clothing and footwear & 98.0 & 98.0 & 97.2 & 97.9 & 97.3 \\
\hline Housing & 103.6 & 103.6 & 102.8 & 103.7 & 104.8 \\
\hline Fuel and light & 100.5 & 100.2 & 107.7 & 126.1 & 137.8 \\
\hline Household furniture and supplies & 92.8 & 97.0 & 102.6 & 99.6 & 101.2 \\
\hline Medical care and expenses & 104.2 & 106.7 & 106.3 & 107.7 & 107.7 \\
\hline Transport and communication & 100.1 & 103.3 & 105.3 & 103.0 & 114.8 \\
\hline Education & 121.7 & 121.7 & 121.8 & 123.7 & 130.1 \\
\hline Personal services & 99.0 & 96.6 & 96.6 & 101.3 & 106.0 \\
\hline \multirow[t]{2}{*}{ Miscellaneous } & 111.4 & 109.8 & 113.3 & 114.3 & 117.5 \\
\hline & \multicolumn{5}{|c|}{ (Percentage change) } \\
\hline End of period & 0.5 & 2.7 & 1.7 & 3.9 & 4.8 \\
\hline Food & -2.0 & 4.5 & 2.0 & 6.0 & 5.7 \\
\hline Alcoholic beverages and tobacco & 2.8 & -1.8 & 3.9 & 4.3 & 2.6 \\
\hline Clothing and footwear & 0.0 & 0.0 & -0.8 & 0.7 & -0.6 \\
\hline Housing & 2.5 & 0.0 & -0.8 & 0.9 & 1.1 \\
\hline Fuel and light & 0.5 & -0.3 & 7.5 & 17.1 & 9.3 \\
\hline Household furniture and supplies & -3.6 & 4.5 & 5.8 & -2.9 & 1.6 \\
\hline Medical care and expenses & 0.0 & 2.4 & -0.4 & 1.3 & 0.0 \\
\hline Transport and communication & 0.0 & 3.2 & 1.9 & -2.2 & 11.5 \\
\hline Education & 24.7 & 0.0 & 0.1 & 1.6 & 5.2 \\
\hline Personal services & 1.1 & -2.4 & 0.0 & 4.9 & 4.6 \\
\hline Miscellaneous & 0.1 & -1.4 & 3.2 & 0.9 & 2.8 \\
\hline
\end{tabular}

Sources: Statistical Office, Central Planning Division, Ministry of Finance and Planning. 
Table 9. St. Vincent and the Grenadines: Summary of Consolidated Public Sector Operations

\begin{tabular}{|c|c|c|c|c|c|}
\hline & 2002 & 2003 & 2004 & 2005 & $\begin{array}{r}\text { Est. } \\
2006 \\
\end{array}$ \\
\hline & \multicolumn{5}{|c|}{ (In millions of Eastern Caribbean dollars) } \\
\hline Total revenue and grants & 345 & 348 & 358 & 381 & 466 \\
\hline Current revenue & 333 & 343 & 349 & 362 & 429 \\
\hline Central government current revenue & 302 & 318 & 322 & 336 & 399 \\
\hline Operating balance $1 /$ & 32 & 25 & 27 & 26 & 30 \\
\hline Capital revenue (land sales only) & 1 & 1 & 1 & 1 & 1 \\
\hline Grants & 11 & 5 & 8 & 17 & 26 \\
\hline Total expenditure & 375 & 379 & 434 & 472 & 547 \\
\hline Current expenditure & 276 & 276 & 290 & 322 & 356 \\
\hline Capital & 99 & 104 & 144 & 149 & 191 \\
\hline Central government & 63 & 82 & 80 & 97 & 118 \\
\hline National Insurance Services & 6 & 0 & 6 & 6 & 6 \\
\hline Rest of the public sector $1 /$ & 30 & 21 & 58 & 47 & 67 \\
\hline Overall balance (above the line) & -30 & -31 & -76 & -91 & -81 \\
\hline Other $2 /$ & 27 & 0 & 0 & 0 & 0 \\
\hline Overall balance (cash basis) & -58 & -31 & -76 & -91 & -81 \\
\hline \multicolumn{6}{|l|}{ Of which } \\
\hline Savings & 57 & 67 & 59 & 40 & 73 \\
\hline Primary balance & -30 & -2 & -45 & -48 & -31 \\
\hline Identified financing & 58 & 31 & 76 & 91 & 81 \\
\hline Net external financing & 3 & 47 & 71 & 64 & 3 \\
\hline Disbursements & 22 & 69 & 105 & 102 & 47 \\
\hline Amortization & 19 & 22 & 35 & 38 & 44 \\
\hline Change in government foreign assets & 0 & 2 & 2 & 0 & 0 \\
\hline Net domestic financing & 53 & -32 & 4 & 27 & 78 \\
\hline Banking system & 31 & -28 & 19 & 62 & 1 \\
\hline Exceptional financing & 1 & 1 & 0 & 0 & 0 \\
\hline \multirow[t]{2}{*}{ Sale of assets } & 1 & 14 & 0 & 0 & 0 \\
\hline & \multicolumn{5}{|c|}{ (In percent of GDP) } \\
\hline Total revenue and grants & 35.0 & 33.7 & 32.0 & 32.2 & 35.0 \\
\hline Current revenue & 33.8 & 33.2 & 31.2 & 30.7 & 32.3 \\
\hline Total expenditure & 38.0 & 36.8 & 38.8 & 39.9 & 41.1 \\
\hline Current expenditure & 28.0 & 26.7 & 26.0 & 27.3 & 26.8 \\
\hline Capital & 10.0 & 10.0 & 12.9 & 12.6 & 14.3 \\
\hline Overall balance (above the line) & -3.1 & -3.0 & -6.8 & -7.7 & -6.3 \\
\hline Overall balance (cash basis) & -5.9 & -3.0 & -6.8 & -7.7 & -6.3 \\
\hline Savings & 5.8 & 6.5 & 5.3 & 3.4 & 5.5 \\
\hline Primary balance & -3.1 & -0.2 & -4.0 & -4.0 & -2.3 \\
\hline Net external financing & 0.3 & 4.5 & 6.3 & 5.4 & 0.3 \\
\hline Net domestic financing & 5.4 & -3.1 & 0.3 & 2.3 & 6.0 \\
\hline \multicolumn{6}{|l|}{ Memorandum items: } \\
\hline Gross public sector debt, percent of GDP 3/ & 70.5 & 72.3 & 77.0 & 80.7 & 78.0 \\
\hline GDP at market prices, EC\$ millions & 986 & 1032 & 1118 & 1182 & 1330 \\
\hline
\end{tabular}

Sources: Ministry of Finance and Planning; and Fund staff estimates.

1/ Operating balances for the National Insurance Services and ten nonfinancial public enterprises.

2/ The difference between the overall balance as measured from above the line and from below the line. $3 /$ Net of intra-public sector debt (mainly central government debt to the NIS). 
Table 10. St. Vincent and the Grenadines: Summary of Central Government Operations

\begin{tabular}{|c|c|c|c|c|c|}
\hline & 2002 & 2003 & 2004 & 2005 & $\begin{array}{r}\text { Est. } \\
2006 \\
\end{array}$ \\
\hline & \multicolumn{5}{|c|}{ (In millions of Eastern Caribbean dollars) } \\
\hline Total revenue and grants & 313 & 324 & 331 & 354 & 409 \\
\hline Current revenue & 302 & 318 & 322 & 336 & 399 \\
\hline Tax revenue & 260 & 260 & 274 & 308 & 363 \\
\hline Income & 88 & 77 & 79 & 90 & 99 \\
\hline Property & 3 & 3 & 3 & 3 & 3 \\
\hline Goods and services & 43 & 47 & 54 & 74 & 98 \\
\hline International trade transactions & 127 & 133 & 138 & 142 & 163 \\
\hline Nontax revenue & 42 & 58 & 48 & 28 & 36 \\
\hline Capital revenue & 1 & 1 & 1 & 1 & 1 \\
\hline Grants & 11 & 5 & 8 & 17 & 9 \\
\hline Total expenditure & 336 & 358 & 370 & 419 & 474 \\
\hline Current expenditure & 276 & 276 & 290 & 322 & 356 \\
\hline Wages and salaries $1 /$ & 143 & 139 & 147 & 159 & 171 \\
\hline Other goods and services & 64 & 61 & 67 & 68 & 77 \\
\hline Interest payments & 26 & 28 & 28 & 35 & 43 \\
\hline Domestic & 15 & 17 & 13 & 15 & 20 \\
\hline Foreign & 11 & 11 & 14 & 20 & 23 \\
\hline Central government transfers & 43 & 48 & 49 & 61 & 65 \\
\hline Capital expenditure & 63 & 82 & 80 & 97 & 118 \\
\hline Net lending & -4 & 0 & 0 & 0 & 0 \\
\hline Overall balance & -22 & -34 & -39 & -65 & -65 \\
\hline Other $2 /$ & 19 & 0 & 0 & 0 & 0 \\
\hline Overall balance & -41 & -34 & -39 & -65 & -65 \\
\hline \multicolumn{6}{|l|}{ Of which } \\
\hline Savings & 26 & 42 & 32 & 14 & 43 \\
\hline Primary balance & -15 & -7 & -12 & -30 & -22 \\
\hline \multicolumn{6}{|l|}{ Financing } \\
\hline External (net) & 3 & 46 & 71 & 37 & -9 \\
\hline Drawings & 20 & 65 & 105 & 74 & 35 \\
\hline Amortization & 16 & 19 & 35 & 37 & 44 \\
\hline Change in government foreign assets & 0 & 2 & 2 & 0 & 0 \\
\hline Domestic (net) & 37 & -15 & -33 & 28 & 74 \\
\hline Banking system & 42 & -51 & 16 & 47 & -3 \\
\hline Nonbanking $3 /$ & -5 & 36 & -50 & -20 & 77 \\
\hline \multirow[t]{2}{*}{ Exceptional financing (interest moratorium) } & 1 & 1 & 0 & 0 & 0 \\
\hline & \multicolumn{5}{|c|}{ (In percent of GDP) } \\
\hline Total revenue and grants & 31.7 & 31.3 & 29.6 & 30.0 & 30.8 \\
\hline Current revenue & 30.6 & 30.8 & 28.8 & 28.5 & 30.0 \\
\hline Tax revenue & 26.3 & 25.2 & 24.6 & 26.1 & 27.3 \\
\hline Nontax revenue & 4.3 & 5.7 & 4.2 & 2.4 & 2.7 \\
\hline Capital revenue & 0.1 & 0.1 & 0.1 & 0.1 & 0.1 \\
\hline Grants & 1.1 & 0.4 & 0.7 & 1.5 & 0.7 \\
\hline Total expenditure & 34.0 & 34.7 & 33.1 & 35.5 & 35.7 \\
\hline Current expenditure & 28.0 & 26.7 & 26.0 & 27.3 & 26.8 \\
\hline Wages and salaries $1 /$ & 14.5 & 13.5 & 13.1 & 13.5 & 12.9 \\
\hline Other goods and services & 6.5 & 6.0 & 6.0 & 5.7 & 5.8 \\
\hline Domestic interest payments & 1.5 & 1.6 & 1.2 & 1.3 & 1.5 \\
\hline Foreign interest payments & 1.1 & 1.1 & 1.3 & 1.7 & 1.7 \\
\hline Transfers & 4.4 & 4.6 & 4.4 & 5.1 & 4.9 \\
\hline Capital expenditure & 6.4 & 7.9 & 7.1 & 8.2 & 8.9 \\
\hline Net lending & -0.4 & 0.0 & 0.0 & 0.0 & 0.0 \\
\hline Overall balance & -2.3 & -3.3 & -3.5 & -5.5 & -4.9 \\
\hline Other $2 /$ & 1.9 & 0.0 & 0.0 & 0.0 & 0.0 \\
\hline $\begin{array}{l}\text { Overall balance } \\
\text { Of which }\end{array}$ & -4.2 & -3.3 & -3.5 & -5.5 & -4.9 \\
\hline Savings & 2.6 & 4.1 & 2.9 & 1.2 & 3.2 \\
\hline Primary balance & -1.6 & -0.6 & -1.1 & -2.5 & -1.7 \\
\hline \multicolumn{6}{|l|}{ Financing } \\
\hline External (net) & 0.3 & 4.5 & 6.3 & 3.1 & -0.7 \\
\hline Domestic (net) & 3.7 & -1.5 & -3.0 & 2.4 & 5.6 \\
\hline
\end{tabular}

Sources: Ministry of Finance and Planning; and Fund staff estimates.

$1 /$ Including employer's contribution to the National Insurance Services (NIS).

2/ The difference between the overall balance as measured from above the line and from below the line.

$3 /$ Including bond issues purchased by the private sector and the change in domestic sinking fund holdings. 
Table 11. St. Vincent and the Grenadines: Central Government Revenues and Grants

\begin{tabular}{|c|c|c|c|c|c|}
\hline & 2002 & 2003 & 2004 & 2005 & $\begin{array}{r}\text { Est. } \\
2006 \\
\end{array}$ \\
\hline & \multicolumn{5}{|c|}{ (In millions of Eastern Caribbean dollars) } \\
\hline Total revenue and grants & 313 & 324 & 331 & 354 & 409 \\
\hline Current revenue & 302 & 318 & 322 & 336 & 399 \\
\hline Tax revenue & 260 & 260 & 274 & 308 & 363 \\
\hline Income & 88 & 77 & 79 & 90 & 99 \\
\hline Individual & 35 & 35 & 37 & 41 & 49 \\
\hline Corporate & 45 & 38 & 37 & 44 & 43 \\
\hline Withholding & 8 & 4 & 4 & 4 & 6 \\
\hline Property & 3 & 3 & 3 & 3 & 3 \\
\hline Goods and services & 43 & 47 & 54 & 74 & 98 \\
\hline Consumption tax (local) & 6 & 6 & 7 & 6 & 7 \\
\hline Excise duties & 2 & 2 & 2 & 2 & 2 \\
\hline Commercial bank interest levy & 8 & 9 & 9 & 8 & 9 \\
\hline Licenses & 13 & 13 & 18 & 18 & 24 \\
\hline Other & 14 & 18 & 18 & 39 & 56 \\
\hline International trade and transactions & 127 & 133 & 138 & 142 & 163 \\
\hline Import duties & 26 & 29 & 30 & 33 & 35 \\
\hline Consumption duties (imports) & 78 & 79 & 81 & 80 & 94 \\
\hline Travel tax & 1 & 1 & 1 & 1 & 2 \\
\hline Customs service charge & 18 & 19 & 21 & 22 & 26 \\
\hline Other & 4 & 4 & 5 & 6 & 6 \\
\hline Nontax revenue & 42 & 58 & 48 & 28 & 36 \\
\hline Departmental enterprises & 0 & 0 & 0 & 0 & 0 \\
\hline Dividends & 0 & 0 & 0 & 0 & 0 \\
\hline ECCB profits & 0 & 0 & 0 & 0 & 0 \\
\hline Interest and rent & 10 & 15 & 7 & 6 & 5 \\
\hline Fees, fines, and sales & 16 & 15 & 13 & 15 & 17 \\
\hline Other & 17 & 29 & 27 & 11 & 14 \\
\hline Capital revenue (land sales only) & 1 & 1 & 1 & 1 & 1 \\
\hline \multirow[t]{2}{*}{ Grants } & 11 & 5 & 8 & 17 & 9 \\
\hline & \multicolumn{5}{|c|}{ (In percent of GDP) } \\
\hline Total revenue and grants & 31.7 & 31.3 & 29.6 & 30.0 & 30.8 \\
\hline Current revenue & 30.6 & 30.8 & 28.8 & 28.5 & 30.0 \\
\hline Tax revenue & 26.3 & 25.2 & 24.5 & 26.1 & 27.3 \\
\hline Income & 8.9 & 7.5 & 7.1 & 7.6 & 7.4 \\
\hline Property & 0.3 & 0.3 & 0.2 & 0.2 & 0.2 \\
\hline Goods and services & 4.3 & 4.6 & 4.9 & 6.2 & 7.4 \\
\hline International trade transactions & 12.8 & 12.9 & 12.4 & 12.0 & 12.3 \\
\hline Nontax revenue & 4.3 & 5.7 & 4.3 & 2.4 & 2.7 \\
\hline Capital revenue (land sales only) & 0.1 & 0.1 & 0.1 & 0.1 & 0.1 \\
\hline Grants & 1.1 & 0.4 & 0.7 & 1.5 & 0.7 \\
\hline
\end{tabular}

Sources: Ministry of Finance and Planning; and Fund staff estimates. 
Table 12. St. Vincent and the Grenadines: Central Government Expenditure and Net Lending

\begin{tabular}{|c|c|c|c|c|c|}
\hline & 2002 & 2003 & 2004 & 2005 & $\begin{array}{r}\text { Est. } \\
2006\end{array}$ \\
\hline & \multicolumn{5}{|c|}{ (In millions of Eastern Caribbean dollars) } \\
\hline Total expenditure and net lending & 336 & 358 & 370 & 419 & 474 \\
\hline Current expenditure & 276 & 276 & 290 & 322 & 356 \\
\hline Wages and salaries $1 /$ & 143 & 139 & 147 & 159 & 171 \\
\hline Salaries & 124 & 121 & 126 & 136 & 149 \\
\hline Wages & 14 & 14 & 17 & 18 & 19 \\
\hline NIS contribution & 4 & 5 & 4 & 5 & 4 \\
\hline Other goods and services & 64 & 61 & 67 & 68 & 77 \\
\hline Interest payments & 26 & 28 & 28 & 35 & 43 \\
\hline Domestic & 15 & 17 & 13 & 15 & 20 \\
\hline Foreign & 11 & 11 & 14 & 20 & 23 \\
\hline Central government transfers & 43 & 48 & 49 & 61 & 65 \\
\hline Households & 25 & 9 & 0 & 44 & ... \\
\hline Capital expenditure & 63 & 82 & 80 & 97 & 118 \\
\hline \multirow[t]{2}{*}{ Net lending } & -4 & 0 & 0 & 0 & 0 \\
\hline & \multicolumn{5}{|c|}{ (In percent of GDP) } \\
\hline Total expenditure & 34.0 & 34.7 & 33.1 & 35.5 & 35.7 \\
\hline Current expenditure & 28.0 & 26.7 & 26.0 & 27.3 & 26.8 \\
\hline Wages and salaries $1 /$ & 14.5 & 13.5 & 13.1 & 13.5 & 12.9 \\
\hline Salaries & 12.6 & 11.7 & 11.2 & 11.5 & 11.2 \\
\hline Wages & 1.4 & 1.3 & 1.5 & 1.5 & 1.4 \\
\hline NIS contribution & 0.4 & 0.4 & 0.4 & 0.4 & 0.3 \\
\hline Other goods and services & 6.5 & 6.0 & 6.0 & 5.7 & 5.8 \\
\hline Interest payments & 2.6 & 2.7 & 2.5 & 3.0 & 3.2 \\
\hline Domestic & 1.5 & 1.6 & 1.2 & 1.3 & 1.5 \\
\hline Foreign & 1.1 & 1.1 & 1.3 & 1.7 & 1.7 \\
\hline Central government transfers & 4.4 & 4.6 & 4.4 & 5.1 & 4.9 \\
\hline Households & 2.6 & 0.9 & 0.0 & 3.7 & $\ldots$ \\
\hline Capital expenditure & 6.4 & 7.9 & 7.1 & 8.2 & 8.9 \\
\hline Net lending & -0.4 & 0.0 & 0.0 & 0.0 & 0.0 \\
\hline
\end{tabular}

Sources: Ministry of Finance and Planning; and Fund staff estimates.

$1 /$ Salaries and wages including social security contributions, commissions, rewards, allowances, and incentives. 
Table 13. St. Vincent and the Grenadines: Central Government Investment Plan (CGIP)

(In millions of Eastern Caribbean dollars)

\begin{tabular}{|c|c|c|c|c|c|c|c|c|c|c|c|c|c|c|c|}
\hline & \multicolumn{3}{|c|}{2002} & \multicolumn{3}{|c|}{2003} & \multicolumn{3}{|c|}{2004} & \multicolumn{3}{|c|}{2005} & \multicolumn{3}{|c|}{$20061 /$} \\
\hline & Budget & Actual & Rate 2/ & Budget & Actual & Rate 2/ & Budget & Actual & Rate 2/ & Budget & Actual & Rate 2/ & Budget & Prel. & Rate 2/ \\
\hline Total investment & 148.4 & 63.3 & 42.7 & 169.2 & 82.0 & 48.5 & 139.8 & 80.2 & 57.4 & 207.0 & 96.7 & 46.7 & 233.9 & 148.2 & 63.4 \\
\hline Agriculture, forestry and fisheries & 10.9 & 4.8 & 43.6 & 13.3 & 15.4 & 116.3 & 15.9 & 8.2 & 51.6 & 12.7 & 5.4 & 42.1 & 7.1 & 0.7 & 9.3 \\
\hline Manufacturing, quarrying and construction & 0.0 & 0.0 & 0.0 & 0.0 & 0.6 & 0.0 & 1.9 & 0.3 & 0.0 & 4.5 & 1.1 & 23.5 & 1.6 & 0.6 & 37.0 \\
\hline Energy & 27.0 & 0.2 & 0.6 & 42.5 & 0.0 & 0.0 & 0.0 & 0.0 & 0.0 & 0.3 & 0.0 & 0.0 & 17.8 & 17.6 & 98.9 \\
\hline Tourism & 0.0 & 1.1 & $\ldots$ & 1.7 & 5.0 & 296.0 & 1.2 & 1.0 & 83.3 & 2.4 & 0.7 & 30.4 & 4.4 & 0.3 & 6.8 \\
\hline Transport and communication & 24.7 & 19.2 & 77.8 & 32.7 & 20.8 & 63.5 & 32.0 & 16.5 & 51.6 & 88.5 & 26.7 & 30.1 & 66.9 & 45.0 & 67.3 \\
\hline Education and training & 21.5 & 6.0 & 28.1 & 15.1 & 7.2 & 47.9 & 24.2 & 13.0 & 53.7 & 39.5 & 25.5 & 64.4 & 34.6 & 19.2 & 55.4 \\
\hline Health and sanitation & 5.4 & 1.9 & 35.2 & 5.1 & 4.4 & 87.2 & 7.4 & 5.3 & 71.6 & 13.9 & 2.2 & 15.6 & 10.0 & 3.1 & 31.1 \\
\hline Water and sewerage & 0.0 & 0.0 & 0.0 & 0.0 & 0.6 & 0.0 & 0.1 & 0.1 & 70.0 & 5.8 & 4.1 & 71.1 & 14.6 & 14.2 & 97.0 \\
\hline Housing & 14.5 & 0.0 & 0.0 & 16.0 & 0.0 & 0.0 & 0.0 & 0.0 & 0.0 & 4.1 & 1.8 & 43.1 & 1.5 & 0.9 & 56.7 \\
\hline Social development & 22.3 & 7.8 & 35.0 & 23.0 & 5.0 & 21.9 & 12.7 & 5.9 & 46.5 & 9.8 & 6.3 & 64.5 & 4.6 & 0.3 & 7.5 \\
\hline Other & 22.2 & 22.4 & 100.7 & 19.9 & 40.9 & 205.8 & 44.4 & 29.9 & 67.4 & 25.5 & 23.1 & 90.5 & 70.8 & 46.3 & 65.4 \\
\hline Total financing & 148.4 & 63.3 & 42.7 & 169.2 & 82.0 & 48.5 & 139.8 & 80.2 & 57.4 & 207.0 & 96.7 & 46.7 & 233.9 & 148.2 & 63.4 \\
\hline External & 73.3 & 30.0 & 40.9 & 103.4 & 70.0 & 67.7 & 65.2 & 113.0 & 173.3 & 145.1 & 91.0 & 62.7 & 144.2 & 93.3 & 64.7 \\
\hline Grants & 33.6 & 10.5 & 31.3 & 46.2 & 5.0 & 10.8 & 35.7 & 7.7 & 21.5 & 54.9 & 17.2 & 31.3 & 44.1 & 17.1 & 38.7 \\
\hline Loans & 39.8 & 19.5 & 49.1 & 57.3 & 65.0 & 113.5 & 29.5 & 105.3 & 356.6 & 90.1 & 73.8 & 81.9 & 100.1 & 76.2 & 76.1 \\
\hline Local (residual) & 75.1 & 33.3 & 44.4 & 65.7 & 12.0 & 18.3 & 74.6 & -32.8 & -44.0 & 61.9 & 5.7 & 9.2 & 89.7 & 54.9 & 61.2 \\
\hline \multicolumn{16}{|l|}{ Memorandum item: } \\
\hline CGIP in percent of GDP & 15.0 & 6.4 & $\ldots$ & 16.5 & 7.9 & $\ldots$ & 12.8 & 7.1 & $\ldots$ & 17.5 & 8.2 & $\ldots$ & 19.8 & 12.5 & $\ldots$ \\
\hline
\end{tabular}

Sources: Ministry of Finance and Planning; and Fund staff estimates.

$1 /$ Includes central government and some government guaranteed projects.

2/ Rate of implementation (in percent). 
Table 14. St. Vincent and the Grenadines: National Insurance Services

\begin{tabular}{|c|c|c|c|c|c|}
\hline & 2002 & 2003 & 2004 & 2005 & $\begin{array}{r}\text { Est. } \\
2006 \\
\end{array}$ \\
\hline & \multicolumn{5}{|c|}{ (In millions of Eastern Caribbean dollars) } \\
\hline Total current receipts & 33.8 & 35.9 & 39.7 & 41.4 & 43.7 \\
\hline Contribution receipts & 18.9 & 20.2 & 21.9 & 23.2 & 25.5 \\
\hline Investment income & 14.9 & 15.7 & 17.8 & 18.2 & 18.2 \\
\hline Total expenditure & 21.1 & 15.7 & 24.0 & 26.3 & 29.1 \\
\hline Benefits and refunds & 11.7 & 12.3 & 14.1 & 16.2 & 18.3 \\
\hline Operating expenditure & 3.3 & 3.3 & 3.6 & 4.5 & 5.1 \\
\hline Capital expenditure & 6.0 & 0.1 & 6.4 & 5.6 & 5.7 \\
\hline Current account balance & 18.8 & 20.3 & 22.1 & 20.7 & 20.3 \\
\hline Overall balance & 12.8 & 20.2 & 15.7 & 15.1 & 14.6 \\
\hline \multicolumn{6}{|l|}{ Financing } \\
\hline Commercial banks & -2.3 & 3.7 & 37.1 & 18.5 & 10.6 \\
\hline Deposits at foreign banks & 15.0 & 5.3 & -0.9 & 3.8 & 0.0 \\
\hline Deposits at local banks & -17.3 & -1.6 & 38.0 & 14.7 & 10.6 \\
\hline \multirow[t]{2}{*}{ Other $1 /$} & -10.5 & -23.9 & -52.8 & -33.6 & -25.2 \\
\hline & \multicolumn{5}{|c|}{ (In percent of GDP) } \\
\hline Total current receipts & 3.4 & 3.5 & 3.6 & 3.5 & 3.3 \\
\hline Contribution receipts & 1.9 & 2.0 & 2.0 & 2.0 & 1.9 \\
\hline Investment income & 1.5 & 1.5 & 1.6 & 1.5 & 1.4 \\
\hline Total expenditure & 2.1 & 1.5 & 2.2 & 2.2 & 2.2 \\
\hline Benefits and refunds & 1.2 & 1.2 & 1.3 & 1.4 & 1.4 \\
\hline Operating expenditure & 0.3 & 0.3 & 0.3 & 0.4 & 0.4 \\
\hline Capital expenditure & 0.6 & 0.0 & 0.6 & 0.5 & 0.4 \\
\hline Current account balance & 1.9 & 2.0 & 2.0 & 1.8 & 1.5 \\
\hline Overall balance & 1.3 & 2.0 & 1.4 & 1.3 & 1.1 \\
\hline Financing & -1.3 & -2.0 & -1.4 & -1.3 & -1.1 \\
\hline Commercial banks & -0.2 & 0.4 & 3.3 & 1.6 & 0.8 \\
\hline Other $1 /$ & -1.1 & -2.3 & -4.7 & -2.8 & -1.9 \\
\hline \multicolumn{6}{|l|}{ Memorandum items: } \\
\hline $\begin{array}{l}\text { Ratio of operating expenses to } \\
\text { contributions, in percent }\end{array}$ & 17.7 & 16.3 & 16.3 & 19.4 & 20.0 \\
\hline Contribution, percent change & 4.8 & 6.9 & 8.3 & 6.1 & 9.9 \\
\hline Benefit and refunds, percent change & 20.3 & 4.7 & 14.5 & 15.0 & 13.0 \\
\hline
\end{tabular}

Sources: Ministry of Finance and Planning; and Fund staff estimates.

1/ Includes investments on the Regional Government Securities Market (RGSM). 
Table 15. St. Vincent and the Grenadines: Public Enterprise Operations (Including Kingstown Board)

\begin{tabular}{|c|c|c|c|c|c|}
\hline & 2002 & 2003 & 2004 & 2005 & $\begin{array}{r}\text { Est. } \\
2006\end{array}$ \\
\hline & \multicolumn{5}{|c|}{ (In millions of Eastern Caribbean dollars) } \\
\hline Total receipts & 120.1 & 133.8 & 145.7 & 164.4 & 183.7 \\
\hline Total expenditure & 136.8 & 150.8 & 198.6 & 205.8 & 197.6 \\
\hline Current expenditure & 107.2 & 129.3 & 140.7 & 159.0 & 173.6 \\
\hline Interest & 1.4 & 1.3 & 3.8 & 8.5 & 7.0 \\
\hline Capital expenditure & 29.6 & 21.5 & 58.0 & 46.8 & 24.0 \\
\hline Overall balance & -16.7 & -17.0 & -52.9 & -41.4 & -13.9 \\
\hline Other $1 /$ & 8.5 & 0.0 & -0.1 & -0.1 & 0.0 \\
\hline Overall balance (cash basis) & -25.2 & -17.0 & -52.8 & -41.3 & -13.9 \\
\hline \multicolumn{6}{|l|}{ Of which } \\
\hline Current balance & 12.9 & 4.5 & 5.0 & 5.4 & 10.1 \\
\hline Primary balance & -23.8 & -15.7 & -49.1 & -32.7 & -6.9 \\
\hline Financing & 25.2 & 17.0 & 52.8 & 41.3 & 13.9 \\
\hline External (net) & -0.5 & 0.5 & 0.0 & 26.6 & 12.0 \\
\hline Drawings & 2.4 & 4.0 & 0.0 & 28.1 & 12.0 \\
\hline Amortization & 2.8 & 3.5 & 0.0 & 1.5 & 0.0 \\
\hline Domestic (net) & 25.1 & 3.0 & 52.8 & 14.7 & 1.9 \\
\hline Banking system & -8.4 & 19.2 & -34.1 & -3.8 & -23.6 \\
\hline Other domestic (net) & 33.4 & -16.2 & 87.0 & 18.5 & 25.4 \\
\hline \multirow[t]{2}{*}{ Sale of assets } & 0.6 & 13.5 & 0.0 & 0.0 & 0.0 \\
\hline & \multicolumn{5}{|c|}{ (In percent of GDP) } \\
\hline Total receipts & 12.2 & 13.0 & 13.0 & 13.9 & 13.8 \\
\hline Total expenditure & 13.9 & 14.6 & 17.8 & 17.4 & 14.9 \\
\hline Current expenditure & 10.9 & 12.5 & 12.6 & 13.5 & 13.0 \\
\hline Interest & 0.1 & 0.1 & 0.3 & 0.7 & 0.5 \\
\hline Capital expenditure & 3.0 & 2.1 & 5.2 & 4.0 & 1.8 \\
\hline Overall balance & -1.7 & -1.6 & -4.7 & -3.5 & -1.0 \\
\hline Other $1 /$ & 0.9 & 0.0 & 0.0 & 0.0 & 0.0 \\
\hline Overall balance (cash basis) & -2.6 & -1.6 & -4.7 & -3.5 & -1.0 \\
\hline \multicolumn{6}{|l|}{ Of which } \\
\hline Current balance & 1.3 & 0.4 & 0.5 & 0.5 & 0.8 \\
\hline Primary balance & -2.4 & -1.5 & -4.4 & -2.8 & -0.5 \\
\hline External (net) & 0.0 & 0.1 & 0.0 & 2.3 & 0.9 \\
\hline Domestic (net) & 2.5 & 0.3 & 4.7 & 1.2 & 0.1 \\
\hline
\end{tabular}

Sources: Ministry of Finance and Planning; and Fund staff estimates.

$1 /$ The difference between the overall balance as measured from above the line and from below the line. 
Table 16. St. Vincent and the Grenadines: Operations of Selected Public Enterprises (In millions of Eastern Caribbean dollars)

\begin{tabular}{|c|c|c|c|c|c|}
\hline & 2002 & 2003 & 2004 & 2005 & $\begin{array}{r}\text { Est. } \\
2006 \\
\end{array}$ \\
\hline & \multicolumn{5}{|c|}{ A. Kingstown Board } \\
\hline Total receipts & 3.1 & 3.0 & 2.5 & 2.4 & 2.8 \\
\hline Total expenditure & 5.0 & 3.4 & 3.6 & 3.4 & 3.4 \\
\hline Current expenditure & 4.0 & 3.4 & 3.6 & 3.4 & 3.4 \\
\hline Capital expenditure & 1.0 & 0.0 & 0.0 & 0.0 & 0.0 \\
\hline Current account balance & -1.0 & -0.4 & -1.1 & -1.0 & -0.6 \\
\hline \multirow[t]{2}{*}{ Overall balance } & -2.0 & -0.4 & -1.1 & -1.0 & -0.6 \\
\hline & \multicolumn{5}{|c|}{ B. Central Water and Sewerage Authority } \\
\hline Total receipts & 10.9 & 20.9 & 17.9 & 19.8 & 18.0 \\
\hline Total expenditure & 9.9 & 30.1 & 24.1 & 31.9 & 25.3 \\
\hline Current expenditure & 7.3 & 18.0 & 17.5 & 19.3 & 17.5 \\
\hline Capital expenditure & 2.7 & 12.2 & 6.6 & 12.6 & 7.8 \\
\hline Current account balance & 3.6 & 2.9 & 0.4 & 0.5 & 0.5 \\
\hline \multirow[t]{2}{*}{ Overall balance } & 1.0 & -9.3 & -6.2 & -12.2 & -7.3 \\
\hline & \multicolumn{5}{|c|}{ C. Port Authority } \\
\hline Total receipts & 11.9 & 23.1 & 10.6 & 11.2 & 15.4 \\
\hline Total expenditure & 9.7 & 11.2 & 11.7 & 12.3 & 14.2 \\
\hline Current expenditure & 8.6 & 10.8 & 11.7 & 12.3 & 13.2 \\
\hline Capital expenditure & 1.1 & 0.4 & 0.0 & 0.0 & 1.0 \\
\hline Current account balance & 3.2 & -1.1 & -1.2 & -1.2 & 2.1 \\
\hline \multirow[t]{2}{*}{ Overall balance } & 2.2 & 11.9 & -1.1 & -1.2 & 1.2 \\
\hline & \multicolumn{5}{|c|}{ D. St. Vincent Electricity Services Ltd. (VINLEC) } \\
\hline Total receipts & 63.5 & 68.7 & 78.9 & 95.4 & 107.4 \\
\hline Total expenditure & 73.5 & 73.0 & 124.2 & 121.5 & 114.5 \\
\hline Current expenditure & 54.7 & 64.9 & 73.1 & 87.5 & 98.5 \\
\hline Capital expenditure & 18.8 & 8.0 & 51.1 & 34.0 & 16.0 \\
\hline Current account balance & 8.7 & 3.7 & 5.7 & 7.8 & 8.7 \\
\hline Overall balance & -10.0 & -4.3 & -45.3 & -26.1 & -7.2 \\
\hline
\end{tabular}

Sources: Ministry of Finance and Planning; and Fund staff estimates. 
Table 17. St. Vincent and the Grenadines: Monetary Survey

\begin{tabular}{|c|c|c|c|c|c|}
\hline & 2002 & 2003 & 2004 & 2005 & 2006 \\
\hline & \multicolumn{5}{|c|}{ (In millions of Eastern Caribbean dollars) } \\
\hline Net foreign assets & 273 & 313 & 427 & 389 & 428 \\
\hline ECCB (imputed reserves) & 142 & 136 & 200 & 186 & 210 \\
\hline Commercial banks & 131 & 177 & 226 & 203 & 218 \\
\hline Net domestic assets & 436 & 409 & 395 & 485 & 511 \\
\hline Public sector credit (net) & -46 & -74 & -54 & 8 & 8 \\
\hline Central government & 86 & 47 & 6 & 74 & 69 \\
\hline $\mathrm{ECCB}$ & 12 & 24 & -31 & -13 & -16 \\
\hline Commercial banks & 74 & 23 & 40 & 87 & 84 \\
\hline Net credit to rest of public sector & -132 & -121 & -63 & -67 & -60 \\
\hline National Insurance Services & -141 & -137 & -100 & -82 & -71 \\
\hline Other & 9 & 16 & 37 & 15 & 11 \\
\hline Credit to private sector & 633 & 637 & 643 & 678 & 775 \\
\hline Net credit to nonbank financial institutions & -54 & -56 & -23 & -32 & -23 \\
\hline Other items (net) & -98 & -99 & -171 & -169 & -249 \\
\hline Broad money & 709 & 722 & 821 & 874 & 940 \\
\hline Money & 239 & 254 & 287 & 328 & 344 \\
\hline Currency in circulation & 54 & 57 & 64 & 74 & 80 \\
\hline Demand deposits & 185 & 197 & 223 & 253 & 264 \\
\hline Quasi-money & 470 & 469 & 534 & 546 & 595 \\
\hline Time deposits & 304 & 321 & 384 & 430 & 477 \\
\hline Savings deposits & 156 & 137 & 124 & 101 & 88 \\
\hline \multirow[t]{2}{*}{ Foreign currency deposits } & 10 & 10 & 26 & 15 & 31 \\
\hline & \multicolumn{5}{|c|}{ (Annual percentage change) } \\
\hline Net foreign assets & 7.4 & 14.9 & 36.2 & -8.9 & 10.2 \\
\hline Credit to private sector & 4.6 & 0.6 & 0.9 & 5.5 & 14.3 \\
\hline Broad money & 8.3 & 1.9 & 13.7 & 6.4 & 7.5 \\
\hline Money & 9.1 & 6.1 & 13.1 & 14.2 & 5.1 \\
\hline \multirow[t]{2}{*}{ Quasi-money $1 /$} & 7.9 & -0.3 & 14.0 & 2.2 & 9.0 \\
\hline & \multicolumn{5}{|c|}{$\begin{array}{c}\text { (Percent contribution compared to M2 } \\
\text { at the beginning of the year) }\end{array}$} \\
\hline Net foreign assets & 2.9 & 5.7 & 15.7 & -4.6 & 4.5 \\
\hline Net domestic assets & 5.4 & -3.9 & -2.0 & 11.0 & 3.0 \\
\hline Net credit to public sector & 4.8 & -4.0 & 2.7 & 7.5 & 0.1 \\
\hline Credit to private sector & 4.3 & 0.6 & 0.8 & 4.3 & 11.1 \\
\hline Net credit to nonbank financial institutions & 0.9 & -0.3 & 4.5 & -1.1 & 1.0 \\
\hline Other assets (net) & -4.6 & -0.2 & -10.0 & 0.3 & -9.2 \\
\hline Broad money & 8.3 & 1.9 & 13.7 & 6.4 & 7.5 \\
\hline \multicolumn{6}{|l|}{ Memorandum items: } \\
\hline Income velocity $2 /$ & 1.4 & 1.4 & 1.4 & 1.4 & 1.4 \\
\hline Deposit interest (average rate per annum) & 4.4 & 4.6 & 3.3 & 2.9 & 2.9 \\
\hline Lending interest rate (average rate per annum) & 11.6 & 11.8 & 9.7 & 9.6 & 9.7 \\
\hline Foreign currency deposits/GDP (in percent) & 1.0 & 1.0 & 2.3 & 1.3 & 2.3 \\
\hline Broad money/GDP (in percent) & 71.9 & 70.0 & 73.5 & 74.0 & 70.6 \\
\hline
\end{tabular}

Sources: ECCB; Ministry of Finance and Planning; and Fund staff estimates.

$1 /$ Including resident foreign currency deposits.

2/ Nominal GDP at market prices divided by liabilities to the private sector. 
Table 18. St. Vincent and Grenadines: Eastern Caribbean Central Bank Operations (In millions of Eastern Caribbean dollars)

\begin{tabular}{|c|c|c|c|c|c|}
\hline & 2002 & 2003 & 2004 & 2005 & 2006 \\
\hline Net (imputed) international reserves & 142 & 136 & 200 & 186 & 210 \\
\hline Net domestic assets & -88 & -80 & -136 & -111 & -130 \\
\hline Net credit to central government & 12 & 24 & -31 & -13 & -16 \\
\hline Treasury bills & 0 & 0 & 0 & 0 & 0 \\
\hline Debentures & 4 & 4 & 4 & 4 & 4 \\
\hline Temporary advances & 8 & 20 & 6 & 5 & 3 \\
\hline Other claims $1 /$ & 0 & 0 & 0 & 0 & 0 \\
\hline Central government deposits (-) & 0 & 0 & -41 & -22 & -23 \\
\hline Net credit to rest of public sector & 0 & 0 & 0 & 0 & 0 \\
\hline Net claims on commercial banks & -99 & -103 & -105 & -99 & -114 \\
\hline Claims & 0 & 0 & 0 & 0 & 0 \\
\hline Loans to commercial banks & 0 & 0 & 0 & 0 & 0 \\
\hline Deposits with commercial banks & 0 & 0 & 0 & 0 & 0 \\
\hline Liabilities & -99 & -103 & -105 & -99 & -114 \\
\hline Currency (cash in vault) & -25 & -28 & -24 & -30 & -21 \\
\hline Statutory reserves & -74 & -75 & -81 & -69 & -94 \\
\hline Fixed deposits & 0 & 0 & 0 & 0 & 0 \\
\hline Liabilities to the private sector & 54 & 57 & 64 & 74 & 80 \\
\hline Currency in circulation & 54 & 57 & 64 & 74 & 80 \\
\hline Total currency issued & 79 & 85 & 89 & 104 & 101 \\
\hline Currency held by banks (-) & -25 & -28 & -24 & -30 & -21 \\
\hline
\end{tabular}

Source: ECCB.

$1 /$ Includes advances to government, plus assets due from participating governments. 
Table 19. St. Vincent and Grenadines: Consolidated Balance Sheet of Commercial Banks (In millions of Eastern Caribbean dollars)

\begin{tabular}{|c|c|c|c|c|c|}
\hline & 2002 & 2003 & 2004 & 2005 & 2006 \\
\hline Net foreign assets & 131 & 177 & 226 & 203 & 218 \\
\hline Foreign assets & 314 & 389 & 438 & 458 & 513 \\
\hline Foreign currency holdings & 6 & 7 & 4 & 4 & 3 \\
\hline Claims on ECCB area banks & 70 & 44 & 67 & 48 & 172 \\
\hline Claims on other banks abroad & 104 & 126 & 63 & 69 & 47 \\
\hline Other 1/ & 134 & 213 & 304 & 337 & 290 \\
\hline Foreign liabilities & -183 & -212 & -212 & -255 & -295 \\
\hline Balances due to ECCB area banks & -26 & -72 & -67 & -105 & -137 \\
\hline Balances due to other banks abroad & -29 & -7 & -18 & -16 & -7 \\
\hline Nonresident deposits & -128 & -134 & -127 & -133 & -148 \\
\hline Net position with ECCB & 104 & 101 & 104 & 98 & 108 \\
\hline Claims on ECCB & 108 & 102 & 106 & 98 & 108 \\
\hline Currency holdings & 25 & 28 & 24 & 30 & 21 \\
\hline Current deposits & 64 & 67 & 82 & 67 & 85 \\
\hline Fixed deposits & 18 & 7 & 0 & 2 & 2 \\
\hline Other & 0 & 0 & 0 & 0 & 0 \\
\hline Liabilities to ECCB & -3 & -2 & -2 & 0 & 0 \\
\hline Net domestic assets & 420 & 388 & 426 & 498 & 533 \\
\hline Net credit to government & 74 & 23 & 40 & 87 & 84 \\
\hline Treasury bills & 44 & 21 & 9 & 23 & 25 \\
\hline Other securities & 16 & 24 & 29 & 55 & 41 \\
\hline Loans and advances & 104 & 98 & 104 & 134 & 149 \\
\hline Deposits (-) & -91 & -120 & -102 & -125 & -130 \\
\hline Net credit to National Insurance Services & -141 & -137 & -100 & -82 & -71 \\
\hline Net credit to nonfinancial public enterprises & 9 & 16 & 37 & 15 & 11 \\
\hline Loans, advances, and investments & 25 & 29 & 50 & 47 & 44 \\
\hline Deposits (-) & -15 & -12 & -13 & -32 & -33 \\
\hline Net credit to nonbank financial institutions & -54 & -56 & -23 & -32 & -23 \\
\hline Loans, advances, and investments & 8 & 10 & 34 & 14 & 13 \\
\hline Deposits (-) & -62 & -66 & -57 & -46 & -36 \\
\hline Credit to private sector $2 /$ & 633 & 637 & 643 & 678 & 775 \\
\hline Interbank float (net) & -3 & -2 & -5 & 3 & 0 \\
\hline Net unclassified assets & -99 & -94 & -166 & -171 & -243 \\
\hline Liabilities to the private sector & 655 & 666 & 757 & 800 & 859 \\
\hline Demand deposits & 185 & 197 & 223 & 253 & 264 \\
\hline Time deposits & 304 & 321 & 384 & 430 & 477 \\
\hline Savings deposits 2/ & 156 & 137 & 124 & 101 & 88 \\
\hline Foreign currency deposits & 10 & 10 & 26 & 15 & 31 \\
\hline
\end{tabular}

Sources: ECCB; and Fund staff estimates.

$1 /$ Includes foreign investments and loans to nonresidents.

2/ Includes Banana Growers Association's accounts. 
Table 20. St. Vincent and Grenadines: Balance Sheet of Local Commercial Banks (In millions of Eastern Caribbean dollars)

\begin{tabular}{|c|c|c|c|c|c|}
\hline & 2002 & 2003 & 2004 & 2005 & 2006 \\
\hline Net foreign assets & 101 & 175 & 193 & 235 & 234 \\
\hline Foreign assets & 214 & 287 & 314 & 390 & 369 \\
\hline Foreign currency holdings & 4 & 4 & 2 & 3 & 2 \\
\hline Claims on ECCB area banks & 55 & 25 & 23 & 28 & 78 \\
\hline Claims on other banks abroad & 70 & 91 & 38 & 61 & 41 \\
\hline Other $1 /$ & 86 & 167 & 251 & 299 & 247 \\
\hline Foreign liabilities & -114 & -113 & -121 & -155 & -135 \\
\hline Balances due to ECCB area banks & -26 & -49 & -62 & -93 & -81 \\
\hline Balances due to other banks abroad & -27 & -5 & -5 & -5 & -4 \\
\hline Nonresident deposits & -61 & -59 & -55 & -57 & -50 \\
\hline Net position with ECCB & 40 & 60 & 52 & 52 & 55 \\
\hline Claims on ECCB & 44 & 61 & 53 & 52 & 55 \\
\hline Currency holdings & 14 & 17 & 16 & 21 & 14 \\
\hline Statutory reserves & 0 & 0 & 0 & 0 & 0 \\
\hline Current deposits 2/ & 30 & 44 & 38 & 31 & 41 \\
\hline Fixed deposits & 0 & 0 & 0 & 0 & 0 \\
\hline Other & 0 & 0 & 0 & 0 & 0 \\
\hline Liabilities to ECCB & -3 & -2 & -2 & 0 & 0 \\
\hline Net domestic assets & 156 & 81 & 122 & 115 & 154 \\
\hline Net credit to government & 28 & -8 & -20 & 17 & 36 \\
\hline Treasury bills & 12 & 7 & 0 & 12 & 13 \\
\hline Other securities & 12 & 12 & 4 & 20 & 26 \\
\hline Loans and advances & 71 & 68 & 78 & 109 & 128 \\
\hline Deposits (-) & -67 & -95 & -102 & -125 & -130 \\
\hline Net credit to National Insurance Services & -131 & -133 & -95 & -80 & -70 \\
\hline Net credit to nonfinancial public enterprises & 12 & 17 & 37 & 15 & 11 \\
\hline Loans, advances, and investments & 24 & 28 & 50 & 47 & 44 \\
\hline Deposits (-) & -12 & -11 & -13 & -32 & -33 \\
\hline Net credit to nonbank financial institutions & -37 & -38 & -2 & -7 & -4 \\
\hline Loans, advances, and investments & 6 & 9 & 34 & 14 & 13 \\
\hline Deposits (-) & -43 & -46 & -36 & -21 & -17 \\
\hline Credit to private sector $3 /$ & 369 & 342 & 310 & 328 & 365 \\
\hline Interbank float (net) & 2 & 2 & -2 & 4 & 0 \\
\hline Net unclassified assets & $-8 \overline{7}$ & -102 & $-10 \overline{7}$ & -162 & -183 \\
\hline Liabilities to the private sector & 297 & 315 & 366 & 402 & 444 \\
\hline Demand deposits & 58 & 63 & 73 & 86 & 89 \\
\hline Time deposits & 60 & 61 & 56 & 52 & 44 \\
\hline Savings deposits $3 /$ & 176 & 187 & 219 & 259 & 289 \\
\hline Foreign currency deposits & 3 & 4 & 19 & 5 & 22 \\
\hline
\end{tabular}

Sources: ECCB; and Fund staff estimates.

$1 /$ Includes foreign investments and loans to nonresidents.

2/ Statutory reserves are included in current deposits.

3/ Banana Growers Association's loans and deposits are treated as private sector credit and deposit. 
Table 21. St. Vincent and the Grenadines: Consolidated Balance Sheet of Foreign Commercial Banks (In millions of Eastern Caribbean dollars)

\begin{tabular}{|c|c|c|c|c|c|}
\hline & 2002 & 2003 & 2004 & 2005 & 2006 \\
\hline Net foreign assets & 30 & 3 & 34 & 9 & -16 \\
\hline Foreign assets & 99 & 102 & 125 & 108 & 143 \\
\hline Foreign currency holdings & 2 & 3 & 2 & 1 & 1 \\
\hline Claims on ECCB area banks & 15 & 19 & 44 & 62 & 94 \\
\hline Claims on other banks abroad & 34 & 34 & 26 & 8 & 6 \\
\hline Other 1/ & 48 & 46 & 53 & 38 & 43 \\
\hline Foreign liabilities & -69 & -100 & -91 & -99 & -160 \\
\hline Balances due to ECCB area banks & -1 & -22 & -5 & -12 & -56 \\
\hline Balances due to other banks abroad & -1 & -2 & -13 & -11 & -3 \\
\hline Nonresident deposits & -67 & -75 & -73 & -76 & -98 \\
\hline Other & 0 & 0 & 0 & 0 & -2 \\
\hline Net position with ECCB & 64 & 41 & 53 & 47 & 53 \\
\hline Claims on ECCB & 64 & 41 & 53 & 47 & 53 \\
\hline Currency holdings & 12 & 11 & 9 & 8 & 7 \\
\hline Statutory reserves & 0 & 0 & 0 & 0 & 0 \\
\hline Current deposits & 34 & 23 & 44 & 36 & 44 \\
\hline Fixed deposits & 18 & 7 & 0 & 2 & 2 \\
\hline Other & 0 & 0 & 0 & 0 & 0 \\
\hline Liabilities to ECCB & 0 & 0 & 0 & 0 & 0 \\
\hline Net domestic assets & 264 & 307 & 304 & 342 & 379 \\
\hline Net credit to government & 47 & 31 & 60 & 70 & 48 \\
\hline Treasury bills & 33 & 14 & 9 & 11 & 12 \\
\hline Other securities & 4 & 12 & 24 & 35 & 15 \\
\hline Loans and advances & 33 & 30 & 26 & 24 & 21 \\
\hline Deposits (-) & -24 & -25 & 0 & 0 & 0 \\
\hline Net credit to National Insurance Services & -10 & -4 & -5 & -1 & -1 \\
\hline Net credit to nonfinancial public enterprises & -3 & -1 & 0 & 0 & 0 \\
\hline Loans, advances, and investments & 0 & 0 & 0 & 0 & 0 \\
\hline Deposits (-) & -3 & -1 & 0 & 0 & 0 \\
\hline Net credit to nonbank financial institutions & -17 & -18 & -21 & -25 & -19 \\
\hline Loans, advances, and investments & 2 & 2 & 0 & 0 & 0 \\
\hline Deposits (-) & -19 & -20 & -21 & -25 & -19 \\
\hline Credit to private sector $2 /$ & 264 & 296 & 333 & 350 & 411 \\
\hline Interbank float (net) & -5 & -4 & -3 & -2 & 0 \\
\hline Net unclassified assets & -11 & 7 & -59 & -50 & -59 \\
\hline Liabilities to the private sector & 359 & 351 & 391 & 398 & 415 \\
\hline Demand deposits & 127 & 134 & 150 & 167 & 175 \\
\hline Time deposits & 96 & 77 & 69 & 50 & 45 \\
\hline Savings deposits 2/ & 128 & 134 & 165 & 172 & 187 \\
\hline Foreign currency deposits & 7 & 6 & 8 & 9 & 9 \\
\hline
\end{tabular}

Sources: ECCB; and Fund staff estimates.

$1 /$ Includes foreign investments and loans to nonresidents.

2/ Banana Growers Association's loans and deposits are treated as private sector credit and deposit. 
Table 22. St. Vincent and the Grenadines: Distribution of Commercial Bank Lending

\begin{tabular}{|c|c|c|c|c|c|}
\hline & 2002 & 2003 & 2004 & 2005 & 2006 \\
\hline & \multicolumn{5}{|c|}{ (In millions of Eastern Caribbean dollars) } \\
\hline Total & 787 & 783 & 824 & 887 & 1,003 \\
\hline Agriculture and fisheries & 15 & 15 & 13 & 12 & 12 \\
\hline Mining and quarrying & 1 & 1 & 1 & 0 & 0 \\
\hline Manufacturing & 19 & 20 & 17 & 18 & 38 \\
\hline Distributive trades & 88 & 85 & 88 & 99 & 90 \\
\hline Tourism & 30 & 18 & 29 & 29 & 29 \\
\hline Entertainment and catering & 8 & 5 & 5 & 4 & 5 \\
\hline Transport and storage & 21 & 24 & 21 & 19 & 21 \\
\hline Public utilities & 2 & 3 & 5 & 5 & 9 \\
\hline Construction and land development & 22 & 22 & 28 & 22 & 36 \\
\hline Government and statutory bodies & 103 & 99 & 123 & 152 & 164 \\
\hline Professional and other services & 34 & 28 & 28 & 37 & 64 \\
\hline Financial institutions & 11 & 32 & 11 & 8 & 6 \\
\hline Personal & 433 & 430 & 454 & 482 & 530 \\
\hline House and land purchases & 180 & 162 & 200 & 194 & 201 \\
\hline Home construction and renovation & 100 & 122 & 108 & 138 & 160 \\
\hline Durable consumer goods & 21 & 20 & 18 & 19 & 23 \\
\hline \multirow[t]{2}{*}{ Other personal } & 132 & 126 & 129 & 131 & 146 \\
\hline & \multicolumn{5}{|c|}{ (In percent of total loans and advances) } \\
\hline Total & 100 & 100 & 100 & 100 & 100 \\
\hline Agriculture and fisheries & 2 & 2 & 2 & 1 & 1 \\
\hline Mining and quarrying & 0 & 0 & 0 & 0 & 0 \\
\hline Manufacturing & 2 & 3 & 2 & 2 & 4 \\
\hline Distributive trades & 11 & 11 & 11 & 11 & 9 \\
\hline Tourism & 4 & 2 & 3 & 3 & 3 \\
\hline Entertainment and catering & 1 & 1 & 1 & 0 & 1 \\
\hline Transport and storage & 3 & 3 & 3 & 2 & 2 \\
\hline Public utilities & 0 & 0 & 1 & 1 & 1 \\
\hline Construction and land development & 3 & 3 & 3 & 2 & 4 \\
\hline Government and statutory bodies & 13 & 13 & 15 & 17 & 16 \\
\hline Professional and other services & 4 & 4 & 3 & 4 & 6 \\
\hline Financial institutions & 1 & 4 & 1 & 1 & 1 \\
\hline Personal & 55 & 55 & 55 & 54 & 53 \\
\hline House and land purchases & 23 & 21 & 24 & 22 & 20 \\
\hline Home construction and renovation & 13 & 16 & 13 & 16 & 16 \\
\hline Durable consumer goods & 3 & 3 & 2 & 2 & 2 \\
\hline Other personal & 17 & 16 & 16 & 15 & 15 \\
\hline
\end{tabular}

Source: ECCB. 
Table 23. St. Vincent and the Grenadines: Interest Rate Structure of Commercial Banks (In percent per annum)

\begin{tabular}{|c|c|c|c|c|c|}
\hline & 2002 & 2003 & 2004 & 2005 & 2006 \\
\hline & \multicolumn{5}{|c|}{ I. Commercial Banks: Minimum and Maximum Rates } \\
\hline \multicolumn{6}{|l|}{ Deposits } \\
\hline Demand & $3.0-6.5$ & $2.5-5.5$ & 3.6 & 3.6 & 3.6 \\
\hline Savings & $3.0-5.0$ & $3.0-5.0$ & $3.0-4.5$ & $3.0-4.5$ & $3.0-4.5$ \\
\hline \multicolumn{6}{|l|}{ Time } \\
\hline Up to 3 months & $3.1-6.0$ & $2.25-4.0$ & $1.0-3.5$ & $1.0-2.5$ & $1.0-3.5$ \\
\hline Between 3 and 6 months & $2.5-5.0$ & $2.75-4.5$ & $1.0-3.75$ & $1.0-3.25$ & $1.0-3.75$ \\
\hline Between 6 and 12 months & $2.0-7.0$ & $2.0-7.2$ & $1.0-4.25$ & $1.0-3.75$ & $1.0-4.0$ \\
\hline \multicolumn{6}{|l|}{ Lending } \\
\hline Prime rate & $9.0-11.0$ & $9.0-11.0$ & $9.0-11.0$ & $9.0-11.0$ & $9.0-11.0$ \\
\hline \multirow[t]{2}{*}{ Other lending rates $1 /$} & $5.0-21.0$ & $7.5-21.0$ & $3.0-16.5$ & $3.0-16.5$ & $3.5-20.0$ \\
\hline & \multicolumn{5}{|c|}{ II. Commercial Banks: Average Rates } \\
\hline Weighted lending rates 2 / & 11.6 & 11.8 & 9.7 & 9.6 & 9.7 \\
\hline Weighted deposit rates $2 /$ & 4.4 & 4.6 & 3.3 & 2.9 & 2.9 \\
\hline Spread & 7.2 & 7.3 & 6.4 & 6.7 & 6.8 \\
\hline
\end{tabular}

Sources: ECCB; and IMF, International Finance Statistics.

$1 /$ The low end of the range relates to the lowest mortgage lending rates offered by banks.

2/ Based on period averages. 
Table 24. St. Vincent and the Grenadines: Summary Balance of Payments

\begin{tabular}{|c|c|c|c|c|c|}
\hline & 2002 & 2003 & 2004 & 2005 & $\begin{array}{r}\text { Est. } \\
2006 \\
\end{array}$ \\
\hline & \multicolumn{5}{|c|}{ (In millions of U.S. dollars, unless otherwise indicated) } \\
\hline Current account & -42.0 & -79.5 & -102.5 & -99.3 & -120.1 \\
\hline Trade balance & -116.5 & -136.7 & -159.7 & -169.8 & -198.5 \\
\hline Exports, f.o.b. & 41.2 & 40.1 & 39.3 & 42.6 & 41.2 \\
\hline \multicolumn{6}{|l|}{ Of which } \\
\hline Bananas & 15.9 & 11.9 & 13.1 & 11.9 & 12.7 \\
\hline Imports, f.o.b. & 157.8 & 176.8 & 199.0 & 212.4 & 239.7 \\
\hline Service balance & 80.0 & 68.2 & 72.0 & 79.2 & 82.4 \\
\hline Receipts & 137.1 & 132.8 & 145.2 & 158.0 & 170.8 \\
\hline Travel & 91.0 & 91.2 & 95.6 & 103.9 & 113.3 \\
\hline Other nonfactor services & 46.1 & 41.6 & 49.7 & 54.1 & 57.6 \\
\hline Payments & 57.1 & 64.6 & 73.2 & 78.8 & 88.4 \\
\hline Travel & 10.2 & 12.6 & 14.3 & 14.9 & 15.7 \\
\hline Other nonfactor services & 46.9 & 51.9 & 58.9 & 63.9 & 72.7 \\
\hline Balance on goods and nonfactor services & -36.5 & -68.5 & -87.7 & -90.6 & -116.1 \\
\hline Exports of goods and nonfactor services & 178.3 & 172.8 & 184.5 & 200.5 & 212.0 \\
\hline Imports of goods and nonfactor services & 214.8 & 241.4 & 272.2 & 291.2 & 328.1 \\
\hline Income balance & -17.6 & -23.8 & -29.0 & -26.8 & -24.2 \\
\hline Net Interest & -4.6 & -4.8 & -5.4 & -5.5 & -6.9 \\
\hline Net other factor services & -13.0 & -19.1 & -23.6 & -21.3 & -17.2 \\
\hline Credits & 3.4 & 3.8 & 4.9 & 8.4 & 13.6 \\
\hline Interest & 1.2 & 1.3 & 2.5 & 4.9 & 5.8 \\
\hline Other factor services & 2.1 & 2.5 & 2.4 & 3.5 & 7.8 \\
\hline Debits & 21.0 & 27.6 & 33.9 & 35.1 & 37.8 \\
\hline Interest & 5.9 & 6.1 & 7.9 & 10.3 & 12.8 \\
\hline \multicolumn{6}{|l|}{ Of which } \\
\hline On public debt & 4.0 & 4.1 & 5.3 & 5.6 & 7.9 \\
\hline Other factor services & 15.1 & 21.5 & 26.0 & 24.8 & 25.0 \\
\hline Current transfers & 12.1 & 12.9 & 14.1 & 18.1 & 20.2 \\
\hline Net private transfers & 12.9 & 13.9 & 14.5 & 18.4 & 14.3 \\
\hline Net official transfers & -0.8 & -1.0 & -0.4 & -0.3 & 5.8 \\
\hline Capital and financial account & 25.9 & 63.4 & 111.8 & 71.0 & 132.2 \\
\hline Capital account & 10.6 & 14.4 & 18.9 & 14.2 & 8.2 \\
\hline Public sector (net) & 9.2 & 12.8 & 16.2 & 11.6 & 5.6 \\
\hline Private sector (net) & 1.5 & 1.6 & 2.7 & 2.6 & 2.6 \\
\hline Financial account & 15.3 & 49.0 & 92.9 & 56.8 & 124.1 \\
\hline Net Official Borrowing & 2.1 & -1.2 & 13.7 & 25.9 & -3.8 \\
\hline Public sector (net) & 2.1 & -1.2 & 13.7 & 25.9 & -3.8 \\
\hline Drawings & 8.5 & 6.1 & 22.6 & 36.0 & 17.8 \\
\hline Repayments & 6.4 & 7.3 & 8.9 & 10.1 & 21.6 \\
\hline Net private capital & 28.5 & 67.3 & 97.4 & 37.4 & 131.6 \\
\hline Net direct investment & 34.0 & 55.2 & 65.7 & 40.1 & 94.3 \\
\hline Equity capital & 0.0 & 8.7 & 33.1 & 12.1 & 38.2 \\
\hline Retained earnings & 11.5 & 14.6 & 16.1 & 11.6 & 12.9 \\
\hline Land sales & 11.8 & 10.6 & 12.8 & 14.3 & 41.3 \\
\hline Private capital & 10.8 & 21.3 & 3.6 & 2.1 & 1.9 \\
\hline Net other private capital & -5.6 & 12.1 & 31.8 & -2.7 & 37.3 \\
\hline Change in net foreign assets of banks & -15.3 & -17.1 & -18.3 & -6.5 & -3.7 \\
\hline Errors and omissions & 9.4 & 15.6 & 16.1 & 25.4 & 0.0 \\
\hline Overall balance & -6.6 & -0.5 & 25.4 & -2.9 & 12.1 \\
\hline & & & (In percent 0 & & \\
\hline Current account balance & -11.5 & -20.8 & -24.8 & -22.7 & -24.4 \\
\hline Trade deficit & -31.9 & -35.8 & -38.6 & -38.8 & -40.3 \\
\hline Exports & 11.3 & 10.5 & 9.5 & 9.7 & 8.4 \\
\hline Imports & 43.2 & 46.2 & 48.1 & 48.5 & 48.6 \\
\hline Services & 21.9 & 17.8 & 17.4 & 18.1 & 16.7 \\
\hline Travel & 24.9 & 23.8 & 23.1 & 23.7 & 23.0 \\
\hline Capital and financial & 7.1 & 16.6 & 27.0 & 16.2 & 26.8 \\
\hline Official borrowing & 0.2 & 0.2 & 0.2 & 0.2 & 0.2 \\
\hline Net official borrowing & 0.6 & -0.3 & 3.3 & 5.9 & -0.8 \\
\hline Private long term (net) & 7.8 & 17.6 & 23.5 & 8.5 & 26.7 \\
\hline Overall balance & -1.8 & -0.1 & 6.1 & -0.7 & 2.5 \\
\hline External debt & 46.5 & 50.9 & 53.3 & 53.8 & 48.0 \\
\hline Debt service (in percent of exports of goods and services) & 6.3 & 7.1 & 9.8 & 10.7 & 11.8 \\
\hline Merchandise terms of trade $(2000=100)$ & 102.1 & 101.1 & 92.4 & 89.6 & 84.5 \\
\hline Nominal GDP at market prices (US\$) & 365.4 & 382.4 & 414.0 & 437.6 & 492.7 \\
\hline
\end{tabular}

Sources: Ministry of Finance and Planning; ECCB; and Fund staff estimates. 
Table 25. St. Vincent and the Grenadines: Major Exports

\begin{tabular}{|c|c|c|c|c|c|}
\hline & 2002 & 2003 & 2004 & 2005 & $\begin{array}{r}\text { Est. } \\
2006\end{array}$ \\
\hline & \multicolumn{5}{|c|}{ (In millions of U.S. dollars) } \\
\hline Total merchandise exports & 41.2 & 40.1 & 39.3 & 42.6 & 41.2 \\
\hline Re-exports & 3.0 & 7.3 & 3.5 & 6.0 & 4.3 \\
\hline Domestic exports & 38.2 & 32.8 & 35.8 & 36.6 & 39.0 \\
\hline Agricultural & 20.8 & 16.5 & 18.2 & 17.8 & 19.3 \\
\hline Bananas & 15.9 & 11.9 & 13.1 & 11.9 & 12.7 \\
\hline Sweet potatoes & 0.3 & 0.2 & 0.4 & 0.4 & 0.4 \\
\hline Eddoes & 0.8 & 0.8 & 1.0 & 1.1 & 1.2 \\
\hline Dasheen & 1.2 & 1.3 & 1.2 & 1.9 & 2.2 \\
\hline Other agricultural crops $1 /$ & 2.5 & 2.4 & 2.5 & 2.5 & 2.7 \\
\hline Manufactured exports & 14.6 & 13.6 & 14.4 & 15.5 & 18.9 \\
\hline Flour & 4.5 & 4.7 & 4.9 & 5.0 & 6.4 \\
\hline Rice & 4.2 & 3.0 & 2.7 & 3.1 & 3.4 \\
\hline Other & 5.9 & 5.9 & 6.7 & 7.4 & 9.1 \\
\hline \multirow[t]{2}{*}{ Other exports } & 2.8 & 2.7 & 3.2 & 3.4 & 0.8 \\
\hline & \multicolumn{5}{|c|}{ (In percent of total exports) } \\
\hline Re-exports & 7.4 & 18.2 & 8.9 & 14.0 & 10.5 \\
\hline Agricultural & 50.3 & 41.1 & 46.3 & 41.7 & 46.9 \\
\hline Bananas & 38.5 & 29.6 & 33.2 & 27.9 & 30.8 \\
\hline Sweet potatoes & 0.8 & 0.5 & 1.0 & 0.9 & 1.1 \\
\hline Eddoes & 2.0 & 1.9 & 2.5 & 2.5 & 3.0 \\
\hline Dasheen & 2.9 & 3.2 & 3.1 & 4.5 & 5.4 \\
\hline Other agricultural crops $1 /$ & 6.2 & 5.9 & 6.4 & 5.9 & 6.7 \\
\hline Manufactured exports & 35.5 & 34.0 & 36.6 & 36.4 & 46.0 \\
\hline Flour & 11.0 & 11.8 & 12.4 & 11.8 & 15.6 \\
\hline Rice & 10.2 & 7.5 & 6.9 & 7.2 & 8.4 \\
\hline Other & 14.3 & 14.7 & 17.2 & 17.4 & 22.0 \\
\hline Other exports & 6.8 & 6.7 & 8.2 & 7.9 & 1.9 \\
\hline
\end{tabular}

Sources: Ministry of Finance and Planning; ECCB; and Fund staff estimates.

1/ Includes arrowroot starch, coconuts, plantains, tannias, yams, ginger, tobacco, nutmeg, and cut flowers. 
Table 26. St. Vincent and the Grenadines: Value, Volume, and Unit Value of Major Agricultural Exports

(Value in millions of U.S. dollars, volume in thousands of metric tons, and unit price in U.S. dollars per metric ton)

\begin{tabular}{|c|c|c|c|c|c|}
\hline & 2002 & 2003 & 2004 & 2005 & $\begin{array}{r}\text { Est. } \\
2006 \\
\end{array}$ \\
\hline Total merchandise exports & 41.2 & 40.1 & 39.3 & 42.6 & 41.2 \\
\hline Re-exports & 3.0 & 7.3 & 3.5 & 6.0 & 4.3 \\
\hline Domestic exports & 38.2 & 32.8 & 35.8 & 36.6 & 39.0 \\
\hline Agricultural exports & 20.8 & 16.5 & 18.2 & 17.8 & 19.3 \\
\hline \multicolumn{6}{|l|}{ Bananas } \\
\hline Value & 15.9 & 11.9 & 13.1 & 11.9 & 12.7 \\
\hline Volume & 37.6 & 26.7 & 28.1 & 25.2 & 22.7 \\
\hline Unit price & 423 & 444 & 465 & 472 & 558 \\
\hline \multicolumn{6}{|l|}{ Arrowroot starch } \\
\hline Value & 0.01 & 0.01 & 0.12 & 0.24 & 0.09 \\
\hline Volume & 0.00 & 0.00 & 0.05 & 0.06 & 0.02 \\
\hline Unit price & 2,315 & 2,469 & 2,452 & 4,172 & 3,663 \\
\hline \multicolumn{6}{|l|}{ Coconuts } \\
\hline Value & 0.11 & 0.08 & 0.09 & 0.12 & 0.10 \\
\hline Volume & 0.31 & 0.21 & 0.24 & 0.34 & 0.25 \\
\hline Unit price & 367 & 362 & 369 & 362 & 381 \\
\hline \multicolumn{6}{|l|}{ Sweet potatoes } \\
\hline Value & 0.33 & 0.19 & 0.40 & 0.39 & 0.59 \\
\hline Volume & 0.73 & 0.43 & 0.88 & 0.88 & 1.29 \\
\hline Unit price & 455 & 456 & 457 & 437 & 456 \\
\hline \multicolumn{6}{|l|}{ Plantains } \\
\hline Value & 0.86 & 0.72 & 0.92 & 0.92 & 0.96 \\
\hline Volume & 2.11 & 1.77 & 2.26 & 2.26 & 2.37 \\
\hline Unit price & 408 & 407 & 407 & 409 & 405 \\
\hline \multicolumn{6}{|l|}{ Tannias } \\
\hline Value & 0.21 & 0.14 & 0.16 & 0.12 & 0.11 \\
\hline Volume & 0.24 & 0.16 & 0.18 & 0.14 & 0.12 \\
\hline Unit price & 881 & 878 & 878 & 864 & 881 \\
\hline \multicolumn{6}{|l|}{ Eddoes } \\
\hline Value & 0.82 & 0.76 & 0.98 & 1.06 & 1.14 \\
\hline Volume & 1.30 & 1.20 & 1.54 & 1.70 & 1.82 \\
\hline Unit price & 630 & 633 & 634 & 627 & 624 \\
\hline \multicolumn{6}{|l|}{ Dasheen } \\
\hline Value & 1.20 & 1.29 & 1.23 & 1.93 & 2.07 \\
\hline Volume & 1.99 & 2.15 & 2.02 & 3.34 & 3.47 \\
\hline Unit price & 606 & 602 & 611 & 577 & 598 \\
\hline \multicolumn{6}{|l|}{ Flour } \\
\hline Value & 4.52 & 4.74 & 4.89 & 5.01 & 4.98 \\
\hline Volume & 9.23 & 9.94 & 10.55 & 10.52 & 10.46 \\
\hline Unit price & 490 & 477 & 463 & 477 & 476 \\
\hline
\end{tabular}

Sources: Ministry of Finance and Planning; Banana Growers Association; and Fund staff estimates. 
Table 27. St. Vincent and the Grenadines: Exports by Country of Destination

\begin{tabular}{|c|c|c|c|c|c|}
\hline & 2002 & 2003 & 2004 & 2005 & $\begin{array}{r}\text { Est. } \\
2006\end{array}$ \\
\hline & \multicolumn{5}{|c|}{ (In millions of U.S. dollars) } \\
\hline Total exports & 41.2 & 40.1 & 39.3 & 42.6 & 41.2 \\
\hline United Kingdom & 15.2 & 11.2 & 12.3 & 10.7 & 16.5 \\
\hline United States & 2.0 & 4.9 & 1.8 & 3.2 & 1.3 \\
\hline Canada & 0.1 & 0.2 & 0.1 & 0.1 & 0.1 \\
\hline CARICOM countries & 20.0 & 20.1 & 21.1 & 24.7 & 21.1 \\
\hline Antigua & 2.4 & 2.7 & 3.0 & 2.5 & 2.7 \\
\hline Barbados & 4.5 & 4.3 & 4.8 & 5.1 & 3.5 \\
\hline Dominica & 1.3 & 1.4 & 1.5 & 2.9 & 1.4 \\
\hline Guyana & 0.2 & 0.1 & 0.1 & 0.2 & 0.4 \\
\hline Jamaica & 1.7 & 1.0 & 0.8 & 0.8 & 1.2 \\
\hline St. Lucia & 3.5 & 3.9 & 4.2 & 4.4 & 3.6 \\
\hline Trinidad and Tobago & 3.9 & 4.2 & 3.6 & 4.9 & 4.2 \\
\hline Other & 2.5 & 2.5 & 3.0 & 4.1 & 4.0 \\
\hline \multirow[t]{2}{*}{ Other } & 3.9 & 3.6 & 3.9 & 3.9 & 2.0 \\
\hline & \multicolumn{5}{|c|}{ (In percent of total) } \\
\hline Total exports & 100.0 & 100.0 & 100.0 & 100.0 & 100.0 \\
\hline United Kingdom & 37.0 & 27.8 & 31.3 & 25.0 & 40.2 \\
\hline United States & 4.8 & 12.3 & 4.7 & 7.6 & 3.3 \\
\hline Canada & 0.2 & 0.6 & 0.3 & 0.3 & 0.2 \\
\hline CARICOM countries & 48.5 & 50.2 & 53.7 & 58.0 & 51.4 \\
\hline Antigua & 5.7 & 6.8 & 7.7 & 5.8 & 6.6 \\
\hline Barbados & 10.9 & 10.6 & 12.2 & 11.9 & 8.6 \\
\hline Dominica & 3.2 & 3.4 & 3.8 & 6.7 & 3.5 \\
\hline Guyana & 0.4 & 0.3 & 0.3 & 0.4 & 1.0 \\
\hline Jamaica & 4.1 & 2.4 & 2.0 & 1.8 & 3.0 \\
\hline St. Lucia & 8.4 & 9.8 & 10.7 & 10.2 & 8.7 \\
\hline Trinidad and Tobago & 9.5 & 10.5 & 9.2 & 11.5 & 10.2 \\
\hline Other & 6.1 & 6.3 & 7.7 & 9.6 & 9.7 \\
\hline Other & 9.5 & 9.0 & 10.0 & 9.1 & 5.0 \\
\hline
\end{tabular}

Sources: Ministry of Finance and Planning; and Fund staff estimates. 
Table 28. St. Vincent and the Grenadines: Merchandise Imports

\begin{tabular}{|c|c|c|c|c|c|}
\hline & 2002 & 2003 & 2004 & 2005 & $\begin{array}{r}\text { Est. } \\
2006 \\
\end{array}$ \\
\hline & \multicolumn{5}{|c|}{ (In millions of U.S. dollars) } \\
\hline Total merchandise imports & 157.8 & 176.8 & 199.0 & 212.4 & 239.7 \\
\hline Food & 39.0 & 37.7 & 42.6 & 44.2 & 50.2 \\
\hline Beverages and tobacco & 4.2 & 4.8 & 5.7 & 6.4 & 7.3 \\
\hline Crude materials & 6.1 & 6.4 & 8.1 & 6.6 & 7.5 \\
\hline Fuels and lubricants & 15.4 & 19.7 & 24.8 & 33.3 & 37.9 \\
\hline Oils and fats & 0.7 & 0.7 & 0.9 & 0.9 & 1.1 \\
\hline Chemicals & 17.7 & 17.7 & 19.0 & 22.2 & 25.2 \\
\hline Manufactured goods & 32.7 & 39.5 & 45.0 & 45.5 & 51.7 \\
\hline Machinery and transport equipment & 41.7 & 49.9 & 51.4 & 53.1 & 58.8 \\
\hline \multirow[t]{2}{*}{ Other Imports } & 0.4 & 0.3 & 1.5 & 0.1 & $\cdots$ \\
\hline & \multicolumn{5}{|c|}{ (In percent of total imports) } \\
\hline Total merchandise imports & 100.0 & 100.0 & 100.0 & 100.0 & 100.0 \\
\hline Food & 24.7 & 21.3 & 21.4 & 20.8 & 20.9 \\
\hline Beverages and tobacco & 2.7 & 2.7 & 2.9 & 3.0 & 3.1 \\
\hline Crude materials & 3.8 & 3.6 & 4.0 & 3.1 & 3.1 \\
\hline Fuels and lubricants & 9.7 & 11.2 & 12.5 & 15.7 & 15.8 \\
\hline Oils and fats & 0.4 & 0.4 & 0.4 & 0.4 & 0.4 \\
\hline Chemicals & 11.2 & 10.0 & 9.5 & 10.4 & 10.5 \\
\hline Manufactured goods & 20.7 & 22.3 & 22.6 & 21.4 & 21.6 \\
\hline Machinery and transport equipment & 26.4 & 28.2 & 25.8 & 25.0 & 24.5 \\
\hline Other Imports & 0.2 & 0.2 & 0.8 & 0.0 & $\ldots$ \\
\hline
\end{tabular}

Sources: Ministry of Finance and Planning; ECCB; and Fund staff estimates. 
Table 29. St. Vincent and the Grenadines: Imports by Country of Origin

\begin{tabular}{|c|c|c|c|c|c|}
\hline & 2002 & 2003 & 2004 & 2005 & $\begin{array}{r}\text { Est. } \\
2006 \\
\end{array}$ \\
\hline & \multicolumn{5}{|c|}{ (In millions of U.S. dollars) } \\
\hline Total imports, c.i.f. & 157.8 & 176.8 & 199.0 & 212.4 & 239.7 \\
\hline United Kingdom & 15.0 & 14.8 & 23.6 & 22.6 & 23.3 \\
\hline United States & 71.4 & 81.7 & 82.7 & 78.5 & 102.9 \\
\hline Canada & 4.1 & 6.0 & 6.1 & 9.2 & 7.9 \\
\hline Japan & 6.3 & 6.6 & 8.3 & 10.0 & 9.9 \\
\hline Netherlands & 0.9 & 1.6 & 1.4 & 1.3 & 3.3 \\
\hline Germany & 1.8 & 1.2 & 1.3 & 2.5 & 2.8 \\
\hline CARICOM countries & 50.3 & 56.6 & 64.6 & 73.1 & 81.3 \\
\hline Antigua and Barbuda & 0.3 & 0.2 & 0.1 & 0.1 & 0.4 \\
\hline Barbados & 7.1 & 7.6 & 8.9 & 9.5 & 12.1 \\
\hline Dominica & 0.6 & 0.5 & 0.6 & 0.6 & 1.2 \\
\hline Guyana & 2.1 & 1.6 & 2.6 & 1.9 & 6.1 \\
\hline Jamaica & 1.3 & 1.8 & 1.4 & 1.6 & 2.1 \\
\hline St. Lucia & 1.5 & 2.2 & 2.2 & 1.6 & 1.0 \\
\hline Trinidad and Tobago & 36.6 & 41.6 & 48.0 & 56.8 & 56.8 \\
\hline Other & 0.9 & 1.0 & 0.7 & 1.0 & 1.5 \\
\hline \multirow[t]{2}{*}{ Other } & 7.9 & 8.4 & 10.9 & 15.1 & 8.2 \\
\hline & \multicolumn{5}{|c|}{ (In percent of total) } \\
\hline Total imports, c.i.f. & 100.0 & 100.0 & 100.0 & 100.0 & 100.0 \\
\hline United Kingdom & 9.5 & 8.4 & 11.9 & 10.6 & 9.7 \\
\hline United States & 45.2 & 46.2 & 41.6 & 37.0 & 42.9 \\
\hline Canada & 2.6 & 3.4 & 3.1 & 4.3 & 3.3 \\
\hline Japan & 4.0 & 3.7 & 4.2 & 4.7 & 4.1 \\
\hline Netherlands & 0.6 & 0.9 & 0.7 & 0.6 & 1.4 \\
\hline Germany & 1.2 & 0.7 & 0.7 & 1.2 & 1.2 \\
\hline CARICOM countries & 31.9 & 32.0 & 32.4 & 34.4 & 33.9 \\
\hline Antigua and Barbuda & 0.2 & 0.1 & 0.1 & 0.0 & 0.2 \\
\hline Barbados & 4.5 & 4.3 & 4.5 & 4.5 & 5.1 \\
\hline Dominica & 0.4 & 0.3 & 0.3 & 0.3 & 0.5 \\
\hline Guyana & 1.3 & 0.9 & 1.3 & 0.9 & 2.5 \\
\hline Jamaica & 0.8 & 1.0 & 0.7 & 0.8 & 0.9 \\
\hline St. Lucia & 0.9 & 1.2 & 1.1 & 0.8 & 0.4 \\
\hline Trinidad and Tobago & 23.2 & 23.5 & 24.1 & 26.8 & 23.7 \\
\hline Other & 0.6 & 0.6 & 0.4 & 0.5 & 0.6 \\
\hline Other & 5.0 & 4.7 & 5.5 & 7.1 & 3.4 \\
\hline
\end{tabular}

Sources: Ministry of Finance and Planning; and Fund staff estimates. 
Table 30. St. Vincent and the Grenadines: Selected Data on Tourism

\begin{tabular}{|c|c|c|c|c|c|}
\hline & 2002 & 2003 & 2004 & 2005 & $\begin{array}{r}\text { Est. } \\
2006 \\
\end{array}$ \\
\hline Total number of visitors & 247,449 & 241,526 & 261,469 & 256,075 & 306,578 \\
\hline \multicolumn{6}{|l|}{ Of which } \\
\hline Stayovers & 77,622 & 78,535 & 86,721 & 94,966 & 97,432 \\
\hline Air & 90,684 & 92,231 & 99,657 & 104,432 & 106,466 \\
\hline Hotels & 15,637 & 15,772 & 13,600 & 19,092 & 13,537 \\
\hline Private residences and others & 61,985 & 62,515 & 73,121 & 76,366 & 83,895 \\
\hline Excursionists 1/ & 13,062 & 13,944 & 12,936 & 8,974 & 9,034 \\
\hline Sea & 156,765 & 149,295 & 161,812 & 151,643 & 200,112 \\
\hline Cruiseships & 70,314 & 64,965 & 77,585 & 69,753 & 106,474 \\
\hline Yachts & 86,451 & 84,330 & 84,227 & 81,890 & 93,638 \\
\hline Average stay (days/visitors) & 8 & 8 & 8 & 8 & 8 \\
\hline \multicolumn{6}{|l|}{ Of which } \\
\hline Hotels & 10 & 10 & 10 & 10 & 10 \\
\hline Private residences and others & 12 & 12 & 12 & 12 & 12 \\
\hline Yachts & 12 & 12 & 12 & 12 & 12 \\
\hline Excursionists & 2 & 2 & 2 & 2 & 2 \\
\hline Volume of visitors ('000 days) & 2,034 & 2,013 & 2,128 & 2,178 & 2,390 \\
\hline Average daily expenditure (U.S. dollars) & 45 & 45 & 45 & 48 & 47 \\
\hline Hotels & 123 & 125 & 128 & 133 & 136 \\
\hline Private Residences and others & 35 & 35 & 36 & 37 & 38 \\
\hline Yachts & 35 & 35 & 36 & 37 & 38 \\
\hline Excursionists & 35 & 35 & 36 & 37 & 38 \\
\hline Cruiseship and others & 17 & 17 & 17 & 19 & $\ldots$ \\
\hline Tourism expenditure (in millions of US\$) & 91 & 91 & 96 & 104 & 113 \\
\hline Tourism expenditure (in millions of EC $\$$ ) & 246 & 246 & 258 & 281 & 306 \\
\hline
\end{tabular}

Sources: ECCB; Department of Tourism, Tourism Statistical Report; and Fund staff estimates.

$1 /$ Includes visitors whose average length of stay is one to two days. 
Table 31. St. Vincent and the Grenadines: Public Sector External Debt 1/ (In millions of U.S. dollars, unless otherwise stated)

\begin{tabular}{lrrrrr}
\hline & 2002 & 2003 & 2004 & 2005 & 2006 \\
\hline $\begin{array}{l}\text { Public sector external debt 2l } \\
\text { By debtor }\end{array}$ & 170 & 195 & $\mathbf{2 2 1}$ & $\mathbf{2 3 5}$ & $\mathbf{2 3 6}$ \\
$\quad$ Central government & 163 & 180 & 221 & 235 & 231 \\
$\quad$ Public enterprises & 7 & 15 & 0 & 1 & 5 \\
By type of creditor & & & & & \\
$\quad$ Official bilateral & 26 & 29 & 31 & 33 & 30 \\
$\quad$ Official multilateral & 73 & 74 & 75 & 80 & 98 \\
$\quad$ Commercial & 71 & 91 & 115 & 123 & 108 \\
Drawings & 8 & 26 & 39 & 27 & 19 \\
$\quad$ Central government & 7 & 24 & 29 & 31 & 23 \\
$\quad$ Public enterprises & 1 & 1 & 10 & -4 & -4 \\
Debt service & 12 & 13 & 18 & 22 & 28 \\
$\quad$ Amortization & 7 & 8 & 13 & 14 & 16 \\
$\quad$ Interest & 5 & 4 & 6 & 8 & 11 \\
Gross public sector external debt, in percent of GDP & 46.5 & 50.9 & 53.3 & 53.8 & 48.0 \\
& & & & & \\
$\quad$ Total debt service in percent of government revenue & & & & & \\
$\quad$ excluding grants & 10.3 & 10.7 & 15.4 & 17.6 & 18.6 \\
External debt service in percent export of goods and services & 6.3 & 7.1 & 9.8 & 10.7 & 11.8 \\
External debt service in percent of broad money 3/ & 4.4 & 4.7 & 6.1 & 6.8 & 7.9 \\
Effective average external interest rate 4/ & 2.7 & 2.4 & 2.7 & 3.4 & 4.7 \\
\hline
\end{tabular}

Sources: Ministry of Finance and Planning; and Fund staff estimates.

1/ The consolidated public sector includes the central government, the National Insurance Services and the nonfinancial public enterprises.

2/ Includes the assumption of private debt for Ottley Hall shipyard in 1999.

$3 /$ Including foreign currency deposits.

4/ Interest payment as percent of the average of debt stock at beginning and end of period. 